\title{
Diet-induced milk fat depression is associated with alterations in ruminal biohydrogenation pathways and formation of novel fatty acid intermediates in lactating cows
}

\author{
Laura Ventto $^{1}$, Heidi Leskinen ${ }^{1}$, Piia Kairenius ${ }^{1}$, Tomasz Stefański ${ }^{1}$, Ali R. Bayat ${ }^{1}$, Johanna Vilkki ${ }^{2}$ and \\ Kevin J. Shingfield ${ }^{1,3} \dagger$ \\ ${ }^{1}$ Nutritional Physiology, Green Technology, Natural Resources Institute Finland (Luke), FI-31600 Jokioinen, Finland \\ ${ }^{2}$ Animal Genomics, Green Technology, Natural Resources Institute Finland (Luke), FI-31600 Jokioinen, Finland \\ ${ }^{3}$ Institute of Biological, Environmental and Rural Sciences, Aberystwyth University, Aberystwyth SY23 3FL, UK \\ (Submitted 27 June 2016 - Final revision received 14 December 2016 - Accepted 3 January 2017 - First published online 27 February 2017)
}

\section{Abstract}

The biohydrogenation theory of milk fat depression (MFD) attributes decreases in milk fat in cows to the formation of specific fatty acids (FA) in the rumen. Trans-10, cis-12-CLA is the only biohydrogenation intermediate known to inhibit milk fat synthesis, but it is uncertain if increased ruminal synthesis is the sole explanation of MFD. Four lactating cows were used in a $4 \times 4$ Latin square with a $2 \times 2$ factorial arrangement of treatments and 35-d experimental periods to evaluate the effect of diets formulated to cause differences in ruminal lipid metabolism and milk fat synthesis on the flow of FA and dimethyl acetal at the omasum. Treatments comprised total mixed rations based on grass silage with a forage:concentrate ratio of 35:65 or 65:35 containing 0 or $50 \mathrm{~g} / \mathrm{kg}$ sunflower oil (SO). Supplementing the high-concentrate diet with SO lowered milk fat synthesis from $-20 \cdot 2$ to $-31.9 \%$ relative to other treatments. Decreases in milk fat were accompanied by alterations in ruminal biohydrogenation favouring the trans-10 pathway and an increase in the formation of specific intermediates including trans-4 to trans-10-18:1, trans-8, trans-10-CLA, trans-9, cis-11-CLA and trans-10, cis-15-18:2. Flow of trans-10, cis-12-CLA at the omasum was greater on high- than low-concentrate diets but unaffected by SO. In conclusion, ruminal trans-10, cis-12-CLA formation was not increased on a diet causing MFD suggesting that other biohydrogenation intermediates or additional mechanisms contribute to the regulation of fat synthesis in the bovine mammary gland.

Key words: Biohydrogenation: Conjugated fatty acids: Cows: Milk fat: Rumen

Clinical studies have indicated that diet is an important factor in the onset and development of chronic human disease including cancer, CVD, insulin resistance and obesity ${ }^{(1)}$. Ruminant-derived foods are a significant source of fat in the human diet ${ }^{(2)}$, and therefore there has been substantial interest in altering the fatty acid (FA) composition of ruminant-derived foods to lower the incidence of chronic diseases ${ }^{(3)}$. Dietary supplements of oilseeds and plant oils are effective in lowering the concentrations of medium-chain saturates and increasing cis-9-18:1 and PUFA in milk $^{(3)}$, changes that are more aligned with public health recommendations, but often induce milk fat depression (MFD) in lactating cows fed low fibre high-concentrate diets ${ }^{(4-7)}$

Several theories have been proposed to explain diet-induced $\mathrm{MFD}^{(8)}$, that is characterised by decreases in milk fat within a few days, with little or no change in the secretion of milk protein and lactose ${ }^{(9-11)}$. Of these, the biohydrogenation theory of diet-induced $\mathrm{MFD}^{(8)}$ is the most widely accepted, which attributes the causal mechanism to changes in ruminal lipid metabolism leading to the formation of specific biohydrogenation intermediates that directly inhibit milk fat synthesis. Trans-10, cis-12-CLA formed during the isomerisation of $18: 2 n-6$ in the rumen ${ }^{(12)}$ is the only intermediate shown unequivocally to inhibit milk fat synthesis in lactating cows ${ }^{(13)}$. However, increases in milk fat trans-10, cis-12-CLA concentrations on diets causing MFD are often lower than would be expected based on the observed enrichment in milk fat to post-ruminal trans-10, cis-12-CLA infusion, suggesting other biohydrogenation intermediates or other mechanisms may also be involved ${ }^{(10,14)}$. Studies involving abomasal infusion of a mixture of FA have provided evidence to suggest that cis-10, trans-12-CLA ${ }^{(15)}$ and trans-9,

Abbreviations: DMA, dimethyl acetal; FA, fatty acid; FAME, fatty acid methyl ester; FC, forage to concentrate; H, high-concentrate diet containing no added oil; HSO, high-concentrate diet containing $50 \mathrm{~g}$ sunflower oil/kg diet DM; L, low-concentrate diet containing no added oil; LSO, low-concentrate diet containing $50 \mathrm{~g}$ sunflower oil/kg diet DM; MFD, milk fat depression; OBCFA, odd- and branched-chain fatty acids; SO, sunflower oil.

* Corresponding author: H. Leskinen, email heidi.leskinen@luke.fi

$\dagger$ Deceased: the authors wish to dedicate this article to the memory of Professor Kevin J. Shingfield. 
cis-11-CLA ${ }^{(16)}$ may also inhibit milk fat synthesis in the lactating cow. Even though inclusion of plant oils in high-concentrate low-fibre diets typically causes $\mathrm{MFD}^{(4-6)}$ direct measurements of biohydrogenation intermediates formed in the rumen of lactating cows under these circumstances are limited ${ }^{(17)}$

In the present investigation, cows were fed a high- or lowconcentrate diet containing no additional lipid or sunflower oil (SO) to test the hypothesis that increases in ruminal synthesis of CLA isomers with known or putative anti-lipolytic activity explain the decrease in milk fat synthesis on a diet causing MFD. Experimental treatments were formulated to cause varying effects on ruminal lipid metabolism and milk fat synthesis allowing specific alterations in the formation of biohydrogenation intermediates and end products associated with a low fibre high-oil diet causing MFD to be identified. In addition to the detailed characterisation of ruminal biohydrogenation intermediates, the effect of experimental treatments on rumen fermentation, nutrient utilisation, enteric methane production and their relation to rumen microbiome, milk fat quality and mammary transcriptome were evaluated but results are not presented here.

\section{Methods}

\section{Cows, experimental design and treatments}

All experimental procedures were approved by the National Animal Ethics Committee (approval no. ESAVI/794/04.10.03/ 2011) in accordance with the guidelines established by the European Community Council Directive 86/609/EEC ${ }^{(18)}$. Four multiparous Finnish Ayrshire cows (89 (SEM 11.8) d postpartum and 691 (SEM 34.8) kg live weight) fitted with rumen cannulae (i.d. $100 \mathrm{~mm}$; Bar Diamond, Inc.) were used in a $4 \times 4$ Latin square with a $2 \times 2$ factorial arrangement of treatments with $35-\mathrm{d}$ experimental periods. Each period consisted of a 14-d adaptation, 12-d sample collection interval and 9-d washout. Treatments comprised isonitrogenous diets based on grass silage (forage:concentrate (FC) ratio 65:35 and 35:65 on a DM basis, respectively) containing either 0 (treatments low-concentrate diet containing no added oil (L) and high-concentrate diet containing no added oil $(\mathrm{H})$, respectively) or high- or low-concentrate diet containing $50 \mathrm{~g} / \mathrm{kg}$ diet DM of SO (treatments HSO and LSO, respectively). SO (Tuko Logistics Ltd) replaced concentrate ingredients (Table 1). From day 27 until the end of each $35-\mathrm{d}$ period all cows were fed the $\mathrm{L}$ diet to minimise treatment carry-over effects and restore milk fat yield to pre-treatment levels. All experimental diets were offered ad libitum as a total mixed ration and fed in four equal amounts at 06.00, 09.00, 16.30 and 19.30 hours. Diets were prepared as total mixed rations to avoid selection of dietary components and maintain the target FC ratio. Cows were housed in individual tie stalls within a dedicated metabolism unit, with free access to water and a salt block, and milked at 07.00 and 16.45 hours.

\section{Sampling and chemical analysis}

Intake of all cows was measured daily. Representative samples of grass silage, total mixed rations and feed refusals were collected daily from day 21 to day 25 of each experimental period, composited within each period for each cow and stored at $-20^{\circ} \mathrm{C}$. The chemical composition of experimental diets was determined using standard methods ${ }^{(19)}$. Samples of SO were collected over the same interval and analysed for FA content and composition. Daily milk yields of all cows were recorded throughout the experiment, but only measurements from day 22 to day 25 of each experimental period were used for the statistical analysis. Milk samples were collected at each milking, from day 22 to day 25, preserved with Bronopol (Valio Ltd) until milk fat, crude protein (CP) and lactose were predicted by IR spectroscopy (Milko-Scan 133B; Foss Electric). Near IR detection of milk constituents was calibrated using milk samples for which reference measurements had previously been made.

The flow of digesta at the omasum canal was determined using the omasal sampling technique ${ }^{(20)}$ and a triple indigestible marker system using Cr-EDTA, Yb-acetate and indigestible neutral detergent fibre (iNDF) as markers for liquid, small and large particulate phases, respectively ${ }^{(21)}$. Cr-EDTA (1000 g) prepared according to standard methods ${ }^{(22)}$ and $\mathrm{Yb}$-acetate $(5.0 \mathrm{~g})$ obtained from a commercial source (DKSH Nordic A/S) were dissolved in 6 litres of distilled water and infused separately into the rumen at a constant rate $(4.2 \mathrm{ml} / \mathrm{min})$ using a peristaltic pump (Watson-Marlow) starting at 15.00 hours on day 18 of each period. To facilitate rapid equilibration of marker concentrations in the rumen, cows also received priming doses of Cr-EDTA $(1500 \mathrm{~g} / \mathrm{d})$ and $\mathrm{Yb}$-acetate $(7.5 \mathrm{~g} / \mathrm{d})$ at the start of marker administration.

Spot samples $(500 \mathrm{ml})$ of digesta entering the omasal canal were collected four times daily at 3-h intervals from day 22 to day 24. Sampling started at 06.00 hours and was advanced $1 \mathrm{~h}$ each day to cover a 12 - $\mathrm{h}$ period that was considered representative of the entire feeding cycle. After each sampling, digesta was immediately stored at $-20^{\circ} \mathrm{C}$. At the end of the study, samples of digesta were thawed at $20^{\circ} \mathrm{C}$, composited for each cow for each period and separated into large particle, small particle and liquid fractions by filtration and centrifugation ${ }^{(20)}$. Each phase was freeze-dried and stored at $-20^{\circ} \mathrm{C}$, whereas subsamples of each fraction for FA analysis were stored at $-80^{\circ} \mathrm{C}$.

Marker concentrations and chemical composition of omasal digesta were determined using standard methods ${ }^{(20)}$. Digesta flow entering the omasal canal was calculated after mathematical reconstruction according to a triple marker method ${ }^{(20)}$. Marker administration was based on the amounts excreted in faeces. Thereafter, appropriate amounts of freeze-dried digesta fractions were weighed to provide a 10-g composite sample before FA analysis.

Faecal marker excretion was determined by total collection performed over $96 \mathrm{~h}$ starting at 18.00 hours on day 21 of each experimental period. Urine was separated from faeces by means of a light harness and flexible tubing attached to the vulva. Concentrations of iNDF, $\mathrm{Cr}$ and $\mathrm{Yb}$ in faeces were determined using the same methods applied to omasal digesta ${ }^{(20)}$.

\section{Lipid analysis}

Fatty acid methyl esters (FAME) of lipid in SO and freeze-dried samples of silage and concentrates were prepared in a one-step extraction-transesterification procedure using chloroform and 
$2 \%(\mathrm{v} / \mathrm{v})$ sulfuric acid in methanol ${ }^{(23)}$. Feed FA content was determined using tritridecanoin (T-135; Nu-Chek-Prep Inc.) as an internal standard and tripalmitin (T-5888; Sigma-Aldrich) as an external standard ${ }^{(23)}$. Following the addition of an internal standard (tridecanoic acid, N-13A; Nu-Chek-Prep Inc.), the $\mathrm{pH}$ of omasal digesta was adjusted to $2 \cdot 0$ using $2 \mathrm{~mol} / 1$ hydrochloric $\operatorname{acid}^{(23)}$. Lipid in omasal digesta was extracted with a mixture $(3: 2 ; \mathrm{v} / \mathrm{v})$ of hexane and isopropanol and FAME were prepared by a two-step base-acid catalysed procedure using sodium methoxide and $2 \%(\mathrm{v} / \mathrm{v})$ sulfuric acid in methanol as catalysts $^{(24)}$.

FAME were quantified using a gas chromatograph (model 6890N; Agilent Technologies) fitted with a CP-Sil 88 column $(100 \mathrm{~m} \times 0.25 \mathrm{~mm}$ i.d., $0.2 \mu \mathrm{m}$ film thickness; Agilent Techno logies) and flame ionization detector. The total FAME profile in a $2-\mu$ l sample volume at a split ratio of 1:50 was determined using a temperature gradient ${ }^{(23)}$ and $\mathrm{H}_{2}$ as the carrier gas operated at constant pressure $(206.8 \mathrm{kPa})$ and nominal initial flow rate of $2 \cdot 1 \mathrm{ml} / \mathrm{min}$. Individual isomers of $18: 1$ were further resolved in a separate analysis under isothermal conditions at $170^{\circ} \mathrm{C}^{(23)}$. Under these conditions trans-10, cis-15-18: 2 and trans-11, cis-15-18:2 eluted as a single peak. To resolve these isomers, GC analysis was repeated using a SLB-IL111 column $(100 \mathrm{~m} \times 0.25 \mathrm{~mm}$ i.d., $0 \cdot 2 \mu \mathrm{m}$ film thickness; Sigma-Aldrich) and He as a carrier gas (nominal initial flow rate of $1.0 \mathrm{ml} / \mathrm{min}$ ) at constant pressure $264.8 \mathrm{kPa}$ and a temperature gradient (initial oven temperature of $168^{\circ} \mathrm{C}$ maintained for $30 \mathrm{~min}$, increased at a rate of $1^{\circ} \mathrm{C} / \mathrm{min}$ to a final temperature of $200^{\circ} \mathrm{C}$ held for $10 \mathrm{~min}$ ). FAME not available as authentic standards were identified in omasal digesta by GC-MS analysis of FAME and 4,4-dimethyloxazoline derivatives prepared from total FAME according to earlier reports ${ }^{(24,25)}$.

Fatty alcohol chains linked to plasmalogens by an alk-1-enyl (vinyl) ether bond in omasal digesta were analysed as dimethyl acetals (DMA) under the same conditions as FAME. DMA were identified based on GC-MS analysis and comparison with previous reports in the literature ${ }^{(26)}$. The distribution of CLA isomers in omasal digesta was determined by HPLC using four silver-impregnated silica columns (ChromSpher 5 Lipids, $250 \times 4.6 \mathrm{~mm}$ i.d., $5 \mu \mathrm{m}$ particle size; Agilent Technologies) coupled in series and $0.1 \%(\mathrm{v} / \mathrm{v})$ acetonitrile in heptane as the mobile phase ${ }^{(24)}$. Under these conditions, cis-10, trans-12-CLA and trans-10, cis-12-CLA elute as a single peak. Both isomers were resolved by repeating the HPLC analysis using a $2 \%(\mathrm{v} / \mathrm{v})$ acetic acid in heptane mobile phase ${ }^{(15)}$.

\section{Statistical analysis}

The retrospective power analyses were implemented with GLIMMIX procedure of SAS (version 9.3; SAS Institute) according to an approach presented by Stroup ${ }^{(27)}$. The power analysis was performed for milk fat content as the response variable. The targeted treatment means were considered to be $26 \mathrm{~g} / \mathrm{kg}$ for HSO and $40 \mathrm{~g} / \mathrm{kg}$ for the other three treatments. The variance estimates used in the power analysis were obtained from the actual data, as the analysis was implemented retrospectively. The variance estimates were 0.32 for the random cow effect and 19.8 for the residual. This yielded the powers of $75 \%$ for interaction between forage ratio and oil supplementation and $96 \%$ for both HSO $v$. LSO and HSO $v$. H which show that the experimental design was suitable for the designed purpose.

DM intake and the yield of milk and milk constituents recorded during days 22-25 of each experimental period were averaged before statistical analysis. Measurements of intake, milk production and flow of nutrients at the omasum were analysed by ANOVA for a $4 \times 4$ Latin square design with a $2 \times 2$ factorial arrangement of treatments, with a statistical model that included the fixed effects of period, concentrate level, SO supplementation and their interaction, and the random effect of cow using the Mixed procedure of SAS. Least-square means with their standard errors are reported and treatment effects were considered significant at $P<0.05$ and considered a trend at $P=0 \cdot 05-0 \cdot 10$.

\section{Results}

\section{Chemical composition of experimental diets}

Silage was of high quality, both in terms of nutritive value (online Supplementary Table S1) and fermentation characteristics (Table 1). Lipid in grass silage contained relatively high proportions of $16: 0,18: 2 n-6$ and $18: 3 n-3$, whereas cis-9-18:1 and 18:2n-6 predominated in concentrates and SO (online Supplementary Table S1).

\section{Nutrient intake and milk production}

By design, silage DM intake was lower $(P<0.001)$ on $H$ than $\mathrm{L}$ treatments, whereas high-concentrate diets increased $(P<0.01)$ total DM intake (Table 2$)$. SO had no effect $(P>0.05)$ on silage DM intake, but tended to lower $(P=0.09)$ total DM intake. Intakes of organic matter (OM), $\mathrm{CP}$, water-soluble carbohydrate and gross energy were higher $(P<0.01)$, and that of neutral detergent fibre (NDF) and potentially digestible NDF (pdNDF) were lower $(P=0.001)$ for $\mathrm{H}$ than $\mathrm{L}$ treatments. SO tended $(P<0 \cdot 10)$ to lower the intake of $\mathrm{OM}, \mathrm{CP}$, water-soluble carbohydrate and fibre, whereas the decrease in starch intake to $\mathrm{SO}$ was greater $(P<0.05$ for $\mathrm{FC} \times \mathrm{SO}$ interaction) when included in the $\mathrm{L}$ than $\mathrm{H}$ diet (Table 2$)$.

Both dietary $\mathrm{FC}$ ratio $(P<0.05)$ and SO $(P<0.001)$ altered total FA intake (Table 2). Intakes of SFA, MUFA and PUFA were greater on the high- than low-concentrate diets $(P<0.05)$ and increased by SO $(P<0 \cdot 001)$. SO increased $(P<0 \cdot 01)$ the intake of $14: 0,15: 0,16: 0$, cis-6-16:1, cis-9-16:1, 18:0, cis-9-18:1, cis-11-18:1, 18:2n-6, 20:0, cis-11-20:1, 22:0 and $23: 0$ to $30: 0$, but lowered $(P<0.05)$ that of cis-13-22:1. Compared with L treatments, intake of $16: 0$, cis-7-16:1, cis-9-16:1, cis-9-18:1, cis-11-18:1 and 18:2n-6 was higher $(P<0 \cdot 05)$ and $12: 0$, trans-3-16:1, 18:3n-3 and 23:0 to 30:0 ingestion was lower $(P<0 \cdot 01)$ on the $\mathrm{H}$ treatments (Table 2$)$.

Treatments had no effect $(P>0 \cdot 05)$ on the yields of milk, energy corrected milk or lactose (Table 3). However, milk protein output was higher $(P<0 \cdot 01)$ on $\mathrm{H}$ than $\mathrm{L}$ diets. SO decreased milk fat content and secretion when included in the $\mathrm{H}$ (mean responses $-1.28 \%$ and $-392 \mathrm{~g} / \mathrm{d}$, respectively; $P<0.01$ for $\mathrm{FC} \times \mathrm{SO}$ interaction), but not $\mathrm{L} \operatorname{diet}$ (mean responses $+0.25 \%$ and $+26 \mathrm{~g} / \mathrm{d}$, respectively; $P<0.01$ for $\mathrm{FC} \times \mathrm{SO}$ interaction). 
Table 1. Formulation and chemical composition of experimental diets*

\begin{tabular}{|c|c|c|c|c|}
\hline & \multicolumn{4}{|c|}{ Treatment } \\
\hline & L & LSO & $\mathrm{H}$ & HSO \\
\hline \multicolumn{5}{|l|}{ Ingredient (g/kg DM) } \\
\hline Grass silage† & 650 & 650 & 350 & 350 \\
\hline Rolled barley & 55 & 42 & 130 & 116 \\
\hline Ground wheat & 165 & 126 & 390 & 352 \\
\hline Rapeseed expellerł & 100 & 100 & 100 & 100 \\
\hline Urea§ & 0 & 2 & 0 & 2 \\
\hline Sunflower oilll & 0 & 50 & 0 & 50 \\
\hline Vitamin and mineral premix $\boldsymbol{\Psi}$ & 30 & 30 & 30 & 30 \\
\hline \multicolumn{5}{|l|}{ Chemical composition (g/kg DM) ${ }^{\star \star}$} \\
\hline Organic matter & 914 & 915 & 928 & 929 \\
\hline Crude protein & 154 & 153 & 150 & 150 \\
\hline Neutral detergent fibre & 386 & 378 & 267 & 262 \\
\hline Indigestible neutral detergent fibre & $82 \cdot 2$ & $80 \cdot 4$ & $59 \cdot 0$ & $58 \cdot 0$ \\
\hline Water-soluble carbohydrate & 32.6 & 31.0 & 31.6 & $31 \cdot 2$ \\
\hline Starch & 143 & 110 & 318 & 290 \\
\hline Gross energy (MJ/kg DM) & $18 \cdot 6$ & $19 \cdot 7$ & $18 \cdot 8$ & $20 \cdot 1$ \\
\hline \multicolumn{5}{|c|}{$\begin{array}{l}\text { L, low-concentrate diet (forage:concentrate (FC) ratio } 65: 35 \text { on a DM basis) with no additional lipid; LSO, low-concentrate diet (FC ratio } 65: 35) \text { containing } \\
50 \mathrm{~g} \text { sunflower oil/kg diet DM; } \mathrm{H} \text {, high-concentrate diet (FC ratio } 35: 65 \text { ) with no additional lipid; HSO, high-concentrate diet (FC ratio 35:65) containing } \\
50 \mathrm{~g} \text { sunflower oil/kg diet DM. } \\
\text { * Values are means of } n 4 \text { determinations. }\end{array}$} \\
\hline \multicolumn{5}{|c|}{$\begin{array}{l}\dagger \text { Restrictively fermented grass silage prepared from the primary growth of mixed timothy (Phleum pratense) and meadow fescue (Festuca pratensis) } \\
\text { swards (54:46, respectively), grown at Jokioinen }\left(60^{\circ} 49^{\prime} \mathrm{N}, 23^{\circ} 28^{\prime} \mathrm{E}\right) \text { treated with a formic acid-based ensiling additive }(0.76 \text { formic acid and } 0.055 \\
\text { ammonium formate, AIV } 2 \text { Plus; Valio Ltd). Mean fermentation characteristics } \mathrm{pH} 3.95 ; \text { in DM }(\mathrm{g} / \mathrm{kg}) ; \text { lactic acid } 63 \cdot 5 \text {, acetic acid } 22 \cdot 1 \text {, propionic acid } \\
0.18 \text {, formic acid } 19 \cdot 1 \text { and water-soluble carbohydrate } 27 \cdot 0 \text {, soluble } \mathrm{N}(\mathrm{g} / \mathrm{kg} \text { total } \mathrm{N}) 634 \text { and ammonium N (g/kg total } \mathrm{N}) 63 \cdot 9 \text {. Grass silage contained } \\
228 \mathrm{~g} / \mathrm{kg} \text { DM (as fed). }\end{array}$} \\
\hline \multicolumn{5}{|c|}{$\begin{array}{l}\text { ‡ Prepared from rapeseed containing low glucosinolate concentrations (Avena Nordic Grain Ltd). } \\
\text { § Urea (Sigma-Aldrich). }\end{array}$} \\
\hline \multicolumn{5}{|c|}{$\begin{array}{l}\text { II Sunflower oil containing (g/100 g total fatty acids) } 16: 0 \text { (6.14), 18:0 (3.91), cis-9-18:1 (27.9), cis-11-18:1 (0.66) and cis-9, cis-12-18:2 (59-1) as major } \\
\text { components (Tuko Logistics Ltd). }\end{array}$} \\
\hline \multicolumn{5}{|c|}{$\begin{array}{l}\text { II Premix (Onni, Melica Finland Ltd) declared as containing (g/kg) Ca (190), Mg (60), Na (135), Zn (2.19), Mn (0.45), Cu (0.40); (mg/kg), I (55), Co (35), } \\
\text { Se (30), DL-a-tocopherol (550), retinol (66) and cholecalciferol (1). } \\
\text { ** DM content (g/kg fresh weight) of L, LSO, H and HSO treatments 474, 479, } 668 \text { and 672, respectively. }\end{array}$} \\
\hline
\end{tabular}

\section{Nutrient flow at the omasum}

Flow of OM, pdNDF and starch at the omasum $(P<0.05)$ was higher and the amount of volatile fatty acids (VFA) and iNDF was lower $(P<0.05)$ on $\mathrm{H}$ than $\mathrm{L}$ treatments (Table 4$)$. SO decreased $(P<0.05)$ VFA, total $\mathrm{N}$ and non-ammonia $\mathrm{N}$ at the omasum, the magnitude of which tended $(P<0 \cdot 10$ for $\mathrm{FC} \times \mathrm{SO}$ interaction) to be greater for the $\mathrm{H}$ than $\mathrm{L}$ diet.

For both $\mathrm{L}$ and $\mathrm{H}$ diets, SO increased $(P<0 \cdot 001)$ total FA flow at the omasum. However, both dietary FC ratio and SO altered the abundance and relative proportions of specific FA escaping the rumen. Changes to higher amounts of concentrates in the diet were characterised by increases $(P<0.05)$ in unresolved cis-6, cis-7, and trans-11-16:1, 15-oxo-18:0, non-conjugated 18:2 and PUFA, and decreases $(P<0.05)$ in trans-3-16:1, 16-oxo-18:0, total $18: 3,24: 0$, cis-15-24:1, cis-17-26:1 and cis-19-28: 1 at the omasum (Table 4 ).

SO increased $(P<0.05)$ the flow of $16: 0,18: 0,15$-oxo-18:0, trans-18:1, total 18:1, CLA, total 20-C FA, 22:0, $24: 0$, cis-19-28:1, SFA and MUFA, and decreased $(P<0 \cdot 01)$ trans-3-16:1 and total $18: 3$ at the omasum (Table 4). However, supplements of SO resulted in greater increases in trans-9-16:1, 10-oxo-18:0 and 13-oxo-18:0 at the omasum when included in the $\mathrm{H}$ than $\mathrm{L}$ diet, whereas the reverse was true for total cis-18:1 $(P<0.05$ for $\mathrm{FC} \times \mathrm{SO}$ interaction). Furthermore, SO increased 26:0, 28:0 and $30: 0$ when included in the L diet, but decreased the amounts of these FA at the omasum following inclusion in the $\mathrm{H}$ diet $(P<0 \cdot 01$ for $\mathrm{FC} \times \mathrm{SO}$ interaction).

SO resulted in the accumulation of trans-18: 1 isomers in the rumen, with increases in specific isomers being dependent on the dietary FC ratio (Table 5 ). On the $\mathrm{H}$ diet, $\mathrm{SO}$ resulted in a greater increase $(P<0.05$ for $\mathrm{FC} \times \mathrm{SO}$ interaction $)$ in trans- 4 to trans-8-18: 1 and trans-10-18:1 at the omasum compared with the $\mathrm{L}$ diet. In contrast, SO resulted in higher amounts of trans-9 and trans-11-18: 1 at the omasum when included in the $\mathrm{L}$ than $\mathrm{H}$ diet $(P<0.05$ for $\mathrm{FC} \times \mathrm{SO}$ interaction). Irrespective of $\mathrm{FC}$ ratio, SO increased $(P<0.001)$ trans-12 to trans-16-18:1 at the omasum. Trans-10-18:1 was the major 18:1 intermediate on the HSO treatment, whereas trans-11-18:1 was the most abundant on the LSO diet. Furthermore, SO increased $(P<0.05)$ cis-13, cis-15 and cis-16-18:1, and resulted in greater increases of cis-9 and cis-12-18:1 at the omasum when included in the $\mathrm{L}$ than $\mathrm{H}$ diet $(P<0.05$ for $\mathrm{FC} \times \mathrm{SO}$ interaction). Amounts of cis-11, cis-13 and cis-16-18: 1 and trans-5, trans-12 to trans-14$18: 1$ at the omasum were higher $(P \leq 0 \cdot 05)$ on the $\mathrm{H}$ than $\mathrm{L}$ diet.

SO resulted in greater increases in several 18:2 isomers, including cis-6, cis-12-18:2, cis-7, cis-12-18:2, cis-9, trans-1218:2, cis-9, trans-13-18:2, trans-9, cis-12-18:2 and trans-9, trans-12-18: 2 when included in the $\mathrm{H}$ than $\mathrm{L}$ diet $(P<0.05$ for $\mathrm{FC} \times \mathrm{SO}$ interaction; Table 5$)$. Irrespective of dietary FC ratio, SO decreased $(P<0.05)$ cis-12, cis-15-18:2 at the omasum. Increases in the proportion of dietary concentrates decreased 
Table 2. Effect of dietary forage:concentrate (FC) ratio and sunflower oil (SO) on nutrient intake in lactating cows* (Least-square mean values with their pooled standard errors; $n$ 16)

\begin{tabular}{|c|c|c|c|c|c|c|c|c|}
\hline \multirow[b]{2}{*}{ Intake } & \multicolumn{4}{|c|}{ Treatment } & \multirow[b]{2}{*}{ SEM } & \multicolumn{3}{|c|}{$P \dagger$} \\
\hline & L & LSO & $\mathrm{H}$ & HSO & & $\mathrm{FC}$ & so & $\mathrm{FC} \times \mathrm{SO}$ \\
\hline Silage DM $(\mathrm{kg} / \mathrm{d})$ & $12 \cdot 7$ & $12 \cdot 4$ & 8.58 & $7 \cdot 66$ & 0.37 & $<0.001$ & 0.15 & 0.38 \\
\hline $\mathrm{SO}(\mathrm{kg} / \mathrm{d})$ & - & 0.88 & - & 1.00 & 0.034 & $0 \cdot 10$ & $<0.001$ & $0 \cdot 10$ \\
\hline Total DM (kg/d) & $19 \cdot 0$ & $18 \cdot 6$ & 23.3 & $20 \cdot 7$ & 0.78 & $<0.01$ & 0.09 & $0 \cdot 18$ \\
\hline Organic matter $(\mathrm{kg} / \mathrm{d})$ & $17 \cdot 3$ & $16 \cdot 9$ & 21.6 & $19 \cdot 2$ & 0.72 & $<0.01$ & 0.09 & $0 \cdot 18$ \\
\hline Crude protein $(\mathrm{kg} / \mathrm{d})$ & 2.90 & 2.82 & 3.49 & $3 \cdot 10$ & 0.122 & $<0.01$ & 0.09 & 0.22 \\
\hline Neutral detergent fibre $(\mathrm{kg} / \mathrm{d})$ & 7.95 & 7.66 & $6 \cdot 73$ & $5 \cdot 89$ & 0.257 & 0.001 & 0.05 & 0.29 \\
\hline Potentially digestible neutral detergent fibre $(\mathrm{kg} / \mathrm{d})$ & 6.37 & $6 \cdot 14$ & $5 \cdot 32$ & 4.66 & 0.201 & 0.001 & 0.06 & 0.30 \\
\hline Water-soluble carbohydrate $(\mathrm{kg} / \mathrm{d})$ & 0.32 & 0.28 & 0.53 & 0.46 & 0.018 & $<0.001$ & 0.02 & 0.37 \\
\hline Starch $(\mathrm{kg} / \mathrm{d})$ & 2.57 & 1.94 & $7 \cdot 18$ & $5 \cdot 83$ & 0.167 & $<0.001$ & $<0.001$ & 0.04 \\
\hline Total gross energy intake $(\mathrm{MJ} / \mathrm{d})$ & 353 & 365 & 438 & 417 & $16 \cdot 0$ & $<0.01$ & 0.78 & 0.30 \\
\hline \multicolumn{9}{|l|}{ Fatty acids $(\mathrm{g} / \mathrm{d})$} \\
\hline $12: 0$ & 0.38 & 0.37 & 0.30 & 0.29 & 0.022 & $<0.01$ & 0.67 & 0.89 \\
\hline $14: 0$ & 1.23 & 1.78 & 1.35 & 1.91 & 0.068 & 0.10 & $<0.001$ & 0.92 \\
\hline $15: 0$ & 0.43 & 0.57 & 0.49 & 0.62 & 0.030 & 0.12 & $<0.01$ & 0.88 \\
\hline $16: 0$ & $48 \cdot 6$ & $96 \cdot 1$ & 63.5 & 112 & 3.91 & $<0.01$ & $<0.001$ & 0.87 \\
\hline $16: 1$ cis -7 & 0.13 & 0.28 & 0.21 & 0.38 & 0.015 & $<0.001$ & $<0.001$ & 0.81 \\
\hline $16: 1$ cis -9 & $1 \cdot 10$ & 1.69 & $1 \cdot 31$ & 1.95 & 0.073 & 0.02 & $<0.001$ & 0.72 \\
\hline $16: 1$ cis- 11 & 0.07 & 0.06 & 0.09 & 0.08 & 0.010 & 0.08 & 0.41 & 0.93 \\
\hline $16: 1$ trans-3 & 2.79 & $2 \cdot 74$ & 1.89 & 1.68 & 0.084 & $<0.001$ & 0.13 & 0.34 \\
\hline $18: 0$ & 4.95 & 37.3 & 5.97 & 42.5 & 1.46 & 0.06 & $<0.001$ & 0.18 \\
\hline $18: 1$ cis-9 & 64.4 & 293 & 90.8 & 347 & $12 \cdot 3$ & 0.01 & $<0.001$ & 0.28 \\
\hline $18: 1$ cis- 11 & 7.96 & $13 \cdot 1$ & $10 \cdot 3$ & 15.8 & 0.62 & $<0.01$ & $<0.001$ & 0.76 \\
\hline $18: 2$ cis- 9, cis -12 & $92 \cdot 3$ & 578 & 161 & 699 & $23 \cdot 7$ & $<0.01$ & $<0.001$ & 0.28 \\
\hline $18: 3$ cis -9, cis -12, cis -15 & $86 \cdot 7$ & $86 \cdot 1$ & 71.8 & 65.4 & $2 \cdot 56$ & $<0.001$ & 0.18 & 0.26 \\
\hline $20: 0$ & 1.83 & 3.93 & 1.74 & 4.03 & 0.146 & 0.96 & $<0.001$ & 0.50 \\
\hline $20: 1$ cis- 11 & 4.97 & 5.98 & 4.49 & 5.48 & 0.196 & 0.05 & $<0.01$ & 0.97 \\
\hline $22: 0$ & 2.32 & $8 \cdot 15$ & 1.97 & 8.55 & 0.284 & 0.95 & $<0.001$ & 0.21 \\
\hline $22: 1$ cis- 13 & 0.30 & 0.26 & 0.31 & 0.26 & 0.022 & 0.71 & 0.05 & 0.78 \\
\hline$\sum 23: 0-30: 0$ & $6 \cdot 46$ & 8.51 & $5 \cdot 10$ & $7 \cdot 13$ & 0.265 & $<0.01$ & $<0.001$ & 0.99 \\
\hline$\sum$ Other $\ddagger$ & 3.63 & $7 \cdot 72$ & 3.76 & 8.29 & 0.317 & 0.31 & $<0.001$ & 0.52 \\
\hline $\bar{\Sigma}$ SFA & $66 \cdot 7$ & 158 & $80 \cdot 9$ & 178 & $6 \cdot 17$ & 0.02 & $<0.001$ & 0.60 \\
\hline$\sum$ MUFA & 83.5 & 320 & 111 & 375 & $13 \cdot 3$ & 0.02 & $<0.001$ & 0.31 \\
\hline$\sum$ PUFA & 180 & 668 & 234 & 769 & $26 \cdot 0$ & 0.02 & $<0.001$ & 0.37 \\
\hline$\sum$ Fatty acids & 331 & 1146 & 426 & 1323 & $45 \cdot 4$ & 0.02 & $<0.001$ & 0.38 \\
\hline
\end{tabular}

Table 3. Effect of dietary forage:concentrate $(\mathrm{FC})$ ratio and sunflower oil $(\mathrm{SO})$ on milk production in lactating cows* (Least-square mean values with their pooled standard errors; $n$ 16)

\begin{tabular}{|c|c|c|c|c|c|c|c|c|}
\hline & \multicolumn{4}{|c|}{ Treatment } & \multirow[b]{2}{*}{ SEM } & \multicolumn{3}{|c|}{$P+$} \\
\hline & L & LSO & $\mathrm{H}$ & $\mathrm{HSO}$ & & FC & So & $\mathrm{FC} \times \mathrm{SO}$ \\
\hline \multicolumn{9}{|l|}{ Yield } \\
\hline Milk (kg/d) & $26 \cdot 7$ & $25 \cdot 7$ & 29.7 & 28.9 & 2.50 & 0.12 & 0.60 & 0.97 \\
\hline Energy corrected milk $(\mathrm{kg} / \mathrm{d}) \ddagger$ & $26 \cdot 1$ & $25 \cdot 5$ & $30 \cdot 0$ & $25 \cdot 1$ & 1.99 & 0.18 & 0.06 & 0.10 \\
\hline Fat $(\mathrm{g} / \mathrm{d})$ & 1050 & 1076 & 1230 & 838 & 84.6 & 0.55 & $<0.01$ & $<0.01$ \\
\hline Protein $(\mathrm{g} / \mathrm{d})$ & 901 & 823 & 1042 & 1046 & $55 \cdot 2$ & $<0.01$ & 0.42 & 0.37 \\
\hline Lactose $(\mathrm{g} / \mathrm{d})$ & 1161 & 1122 & 1292 & 1257 & 115 & 0.14 & 0.64 & 1.00 \\
\hline \multicolumn{9}{|l|}{ Concentration (\%) } \\
\hline Fat & 3.94 & $4 \cdot 19$ & 4.22 & 2.94 & 0.177 & 0.03 & 0.03 & $<0.01$ \\
\hline Protein & 3.38 & 3.23 & 3.57 & 3.77 & 0.128 & $<0.01$ & 0.77 & 0.11 \\
\hline Lactose & 4.34 & 4.34 & 4.33 & 4.31 & 0.034 & 0.42 & 0.78 & 0.76 \\
\hline
\end{tabular}

L, low-concentrate diet (FC ratio 65:35 on a DM basis) with no additional lipid; LSO, low-concentrate diet (FC ratio 65:35) containing 50 g SO/kg diet DM; $\mathrm{H}$, high-concentrate diet (FC ratio 35:65) with no additional lipid; HSO, high-concentrate diet (FC ratio 35:65) containing 50 g SO/kg diet DM.

* Values represent the mean over days 22-25 of each experimental period.

$\dagger$ Significance of effects due to dietary FC ratio, supplements of $\mathrm{SO}$ and their interaction $(\mathrm{FC} \times \mathrm{SO})$.

$\ddagger$ Energy-corrected milk calculated as milk $(\mathrm{kg} / \mathrm{d}) \times(3.83 \times$ fat $(\%)+2.42 \times$ protein $(\%)+1.654 \times$ lactose $(\%)+2.07) / 3140^{(55)}$ 
Table 4. Effect of dietary forage:concentrate (FC) ratio and sunflower oil (SO) on nutrient flow at the omasum in lactating cows ${ }^{\star}$ (Least-square mean values with their pooled standard errors; $n$ 16)

\begin{tabular}{|c|c|c|c|c|c|c|c|c|}
\hline \multirow[b]{2}{*}{ Flow } & \multicolumn{4}{|c|}{ Treatment } & \multirow[b]{2}{*}{ SEM } & \multicolumn{3}{|c|}{$P \dagger$} \\
\hline & L & LSO & $\mathrm{H}$ & $\mathrm{HSO}$ & & $\mathrm{FC}$ & so & $\mathrm{FC} \times \mathrm{SO}$ \\
\hline $\mathrm{DM}(\mathrm{kg} / \mathrm{d})$ & 13.7 & 13.7 & $15 \cdot 3$ & 13.9 & 0.71 & 0.11 & 0.19 & 0.18 \\
\hline Organic matter $(\mathrm{kg} / \mathrm{d})$ & $10 \cdot 4$ & $10 \cdot 7$ & $12 \cdot 2$ & 11.1 & 0.56 & 0.03 & 0.29 & 0.13 \\
\hline Neutral detergent fibre $(\mathrm{kg} / \mathrm{d})$ & 3.67 & 3.68 & 3.86 & 3.87 & 0.194 & 0.32 & 0.97 & 0.98 \\
\hline Potentially digestible neutral detergent fibre $(\mathrm{kg} / \mathrm{d})$ & $2 \cdot 10$ & $2 \cdot 17$ & 2.46 & 2.65 & 0.140 & 0.02 & 0.37 & 0.68 \\
\hline Indigestible neutral detergent fibre $(\mathrm{kg} / \mathrm{d})$ & 1.58 & 1.52 & 1.40 & 1.22 & 0.060 & $<0.01$ & 0.07 & 0.31 \\
\hline Volatile fatty acids $(\mathrm{kg} / \mathrm{d})$ & 1.67 & 1.58 & 1.62 & 1.22 & 0.104 & 0.04 & 0.02 & 0.09 \\
\hline$N(g / d)$ & 527 & 500 & 651 & 528 & $25 \cdot 3$ & 0.01 & 0.01 & 0.07 \\
\hline Non-ammonia N (g/d) & 524 & 496 & 644 & 526 & $25 \cdot 0$ & 0.01 & 0.01 & 0.08 \\
\hline Starch $(g / d)$ & 367 & 383 & 549 & 621 & $79 \cdot 1$ & 0.01 & 0.49 & 0.66 \\
\hline \multicolumn{9}{|l|}{ Fatty acids (g/d) } \\
\hline $12: 0$ & 0.96 & 0.96 & 1.54 & 1.90 & 0.428 & 0.13 & 0.69 & 0.69 \\
\hline $14: 0$ & 3.46 & 3.48 & 3.97 & 3.15 & 0.482 & 0.86 & 0.44 & 0.42 \\
\hline $16: 0$ & $77 \cdot 7$ & 121 & $98 \cdot 6$ & 126 & 8.69 & 0.15 & $<0.01$ & 0.37 \\
\hline$\sum 16: 1$ & 1.03 & 1.04 & 1.49 & 1.41 & 0.183 & 0.06 & 0.85 & 0.82 \\
\hline $16: 1$ cis-6 + cis- $7+$ trans -11 & 0.17 & 0.23 & 0.38 & 0.38 & 0.036 & 0.001 & 0.42 & 0.41 \\
\hline $16: 1$ cis-9 & 0.43 & 0.45 & 0.78 & 0.74 & 0.143 & 0.06 & 0.93 & 0.82 \\
\hline $16: 1$ trans-3 & 0.41 & 0.32 & 0.32 & 0.24 & 0.022 & $<0.01$ & $<0.01$ & 0.83 \\
\hline $16: 1$ trans -9 & 0.03 & 0.05 & 0.01 & 0.06 & 0.007 & 0.99 & $<0.001$ & 0.04 \\
\hline $18: 0$ & 316 & 858 & 387 & 896 & $60 \cdot 0$ & 0.40 & $<0.001$ & 0.79 \\
\hline 10-oxo-18:0 & 5.07 & 9.74 & $4 \cdot 26$ & 18.6 & 1.940 & 0.08 & $<0.01$ & 0.05 \\
\hline 13-oxo-18:0 & 2.09 & $2 \cdot 31$ & $2 \cdot 17$ & 4.08 & 0.342 & 0.04 & 0.02 & 0.05 \\
\hline 15-oxo-18:0 & 0.25 & 0.34 & 0.30 & 0.62 & 0.059 & 0.03 & 0.01 & 0.11 \\
\hline 16-oxo-18:0 & 0.39 & 0.46 & 0.20 & 0.16 & 0.042 & 0.001 & 0.73 & 0.23 \\
\hline$\sum 18: 1$ cis & $27 \cdot 4$ & 50.5 & 44.8 & 53.6 & 4.40 & 0.01 & 0.001 & 0.04 \\
\hline$\sum 18: 1$ trans & $54 \cdot 0$ & 223 & 69.0 & 280 & $19 \cdot 32$ & 0.10 & $<0.001$ & 0.30 \\
\hline$\sum 18: 1$ & 81.4 & 274 & 114 & 333 & 21.38 & 0.06 & $<0.001$ & 0.52 \\
\hline$\sum$ Non-conjugated $18: 2$ & 21.6 & 24.9 & 31.3 & 35.8 & 2.33 & $<0.01$ & 0.11 & 0.80 \\
\hline$\Sigma \mathrm{CLA}$ & 4.59 & 9.94 & 7.79 & 8.95 & 1.484 & 0.29 & 0.01 & 0.07 \\
\hline$\sum 18: 3$ & 9.47 & $7 \cdot 16$ & 8.27 & $5 \cdot 61$ & 0.501 & 0.02 & 0.001 & 0.71 \\
\hline$\sum 20-C$ fatty acids & $6 \cdot 77$ & 9.86 & 8.01 & $10 \cdot 3$ & 0.492 & 0.11 & 0.001 & 0.41 \\
\hline $22: 0$ & 3.64 & 9.69 & $3 \cdot 21$ & $9 \cdot 14$ & 0.414 & 0.25 & $<0.001$ & 0.88 \\
\hline $24: 0$ & 3.12 & 5.08 & 2.78 & 4.06 & 0.210 & 0.02 & $<0.001$ & 0.16 \\
\hline $24: 1$ cis-15 & 1.08 & 1.07 & 0.95 & 0.81 & 0.058 & $<0.01$ & 0.17 & 0.27 \\
\hline $26: 0$ & 9.55 & 13.9 & 6.98 & $2 \cdot 15$ & 0.577 & $<0.001$ & 0.71 & $<0.001$ \\
\hline $26: 1$ cis- 17 & 0.17 & 0.15 & 0.12 & 0.12 & 0.013 & 0.02 & 0.71 & 0.47 \\
\hline $28: 0$ & 4.55 & $6 \cdot 33$ & 3.34 & 1.84 & 0.253 & $<0.001$ & 0.61 & $<0.001$ \\
\hline $28: 1$ cis- 19 & 0.10 & 0.12 & 0.03 & 0.10 & 0.014 & 0.02 & 0.02 & 0.13 \\
\hline $30: 0$ & $2 \cdot 77$ & 3.64 & 2.02 & 1.48 & 0.150 & $<0.001$ & 0.32 & $<0.01$ \\
\hline$\sum$ Unidentified & $29 \cdot 3$ & $25 \cdot 4$ & $18 \cdot 3$ & 19.5 & 2.64 & 0.02 & 0.63 & 0.36 \\
\hline$\sum$ SFA & 522 & 1120 & 593 & 1129 & 73.2 & 0.60 & $<0.001$ & 0.69 \\
\hline$\sum$ MUFA & 87.5 & 280 & 121 & 340 & 21.7 & 0.06 & $<0.001$ & 0.52 \\
\hline$\sum$ PUFA & 35.7 & $42 \cdot 1$ & 47.6 & 50.5 & 3.92 & 0.02 & 0.15 & 0.57 \\
\hline$\sum$ Fatty acids & 675 & 1467 & 779 & 1539 & $76 \cdot 3$ & 0.25 & $<0.001$ & 0.82 \\
\hline
\end{tabular}

L, low-concentrate diet (FC ratio 65:35 on a DM basis) with no additional lipid; LSO, low-concentrate diet (FC ratio 65:35) containing 50 g SO/kg diet DM; H, high-concentrate diet (FC ratio 35:65) with no additional lipid; HSO, high-concentrate diet (FC ratio $35: 65$ ) containing $50 \mathrm{~g} \mathrm{SO} / \mathrm{kg}$ diet DM.

* Values represent the mean over days 22-24 of each omasal digesta sampling.

† Significance of effects due to dietary FC ratio, supplements of SO and their interaction (FC $\times \mathrm{SO})$.

( $P \leq 0.001)$ trans-11, cis-15-18: 2 and trans-11, trans-15-18:2 and increased $(P<0 \cdot 001)$ cis-9, cis-12-18:2 at the omasum.

Flows of trans-10, cis-12-CLA and trans-10, trans-12-CLA were higher $(P<0.01)$ and that of trans-12, trans-14-CLA was lower $(P<0.05)$ on $\mathrm{H}$ compared with $\mathrm{L}$ treatments (Table 5$)$. SO increased $(P<0.05)$ trans-9, trans-11-CLA and trans-10, trans-12-CLA at the omasum. When included in the L diet, SO increased the flow of cis-10, cis-12-CLA and cis-9, trans-11-CLA, but decreased the amount of both isomers at the omasum when included in the $\mathrm{H}$ diet $(P<0.05$ for $\mathrm{FC} \times \mathrm{SO}$ interaction). Furthermore, SO resulted in larger increases in trans-8, trans-10-CLA flow when included in the $\mathrm{H}$ than $\mathrm{L}$ diet $(P<0.05$ for $\mathrm{FC} \times \mathrm{SO}$ interaction). In contrast, decreases in trans-11, cis-13-CLA to SO were greater when included in the $\mathrm{L}$ than $\mathrm{H}$ diet $(P<0.05$ for $\mathrm{FC} \times \mathrm{SO}$ interaction), whereas the reverse was true for trans-12, cis-14-CLA ( $P=0.05$ for $\mathrm{FC} \times \mathrm{SO}$ interaction). No cis-10, trans-12-CLA was detected in the omasal digesta.

SO lowered $(P<0 \cdot 05)$ cis-9, cis-12, cis-15-18:3 and unresolved trans-9, trans-12, cis-15-18:3 and cis-9, cis-12, trans-15-18:3 at the omasum (Table 5). Flows of cis-9, cis-12, cis-15-18:3, unresolved trans-9, trans-12, cis-15-18:3 and cis-9, cis-12, trans-15-18:3 and cis-9, trans-11, cis-15-18:3 were higher $(P<0.05)$ on $\mathrm{L}$ than $\mathrm{H}$ treatments. SO increased $(P<0 \cdot 05) 20: 0$, cis-9+trans-14-20:1, cis-11-20:1 and trans-13-20:1 and 22:0 at the omasum (online Supplementary Table S2), whereas increases in dietary concentrates resulted in higher $(P \leq 0 \cdot 05)$ amounts of cis-9, cis-11 and trans-11+cis-15-20:1, trans-13-20:1, $20: 2 n-6$ and cis-13-22:1 at the omasum. Including $\mathrm{SO}$ in the $\mathrm{H}$ diet 
Table 5. Effect of dietary forage:concentrate $(\mathrm{FC})$ ratio and sunflower oil (SO) on the flow of 18-carbon unsaturated fatty acids at the omasum in lactating cows*

(Least-square mean values with their pooled standard errors; $n$ 16)

\begin{tabular}{|c|c|c|c|c|c|c|c|c|}
\hline \multirow[b]{2}{*}{ Flow } & \multicolumn{4}{|c|}{ Treatment } & \multirow[b]{2}{*}{ SEM } & \multicolumn{3}{|c|}{$P+$} \\
\hline & $\mathrm{L}$ & LSO & $\mathrm{H}$ & HSO & & $\mathrm{FC}$ & so & $\mathrm{FC} \times \mathrm{SO}$ \\
\hline \multicolumn{9}{|l|}{$18: 1(\mathrm{~g} / \mathrm{d})$} \\
\hline $18: 1$ cis-9 & $18 \cdot 7$ & 30.0 & 29.8 & 30.7 & 3.06 & 0.03 & 0.03 & 0.04 \\
\hline $18: 1 c i s-11$ & 3.90 & 4.90 & 7.07 & $7 \cdot 70$ & 0.973 & 0.02 & 0.40 & 0.84 \\
\hline $18: 1$ cis- 12 & 2.24 & $10 \cdot 4$ & 4.75 & 8.20 & 0.905 & 0.84 & $<0.001$ & 0.03 \\
\hline $18: 1 \mathrm{cis}-13$ & 0.37 & 0.67 & 0.63 & 1.04 & 0.144 & 0.05 & 0.04 & 0.68 \\
\hline $18: 1$ cis- 15 & 1.24 & $2 \cdot 11$ & 1.30 & 3.02 & 0.295 & 0.15 & $<0.01$ & 0.20 \\
\hline $18: 1$ cis-16ł & 0.92 & 2.47 & 1.24 & 2.93 & 0.160 & 0.02 & $<0.001$ & 0.61 \\
\hline $18: 1$ trans -4 & 1.02 & $2 \cdot 70$ & 0.99 & $3 \cdot 61$ & 0.194 & 0.05 & $<0.001$ & 0.04 \\
\hline $18: 1$ trans -5 & 0.54 & 1.52 & 0.54 & $2 \cdot 36$ & 0.130 & 0.02 & $<0.001$ & 0.02 \\
\hline $18: 1$ trans $-6+7+8$ & $2 \cdot 70$ & $12 \cdot 4$ & 3.66 & $17 \cdot 3$ & 0.765 & $<0.01$ & $<0.001$ & 0.04 \\
\hline $18: 1$ trans -9 & 1.68 & $7 \cdot 70$ & 2.28 & $6 \cdot 61$ & 0.345 & 0.45 & $<0.001$ & 0.03 \\
\hline $18: 1$ trans -10 & 2.89 & $20 \cdot 1$ & 7.03 & 133 & $15 \cdot 505$ & $<0.01$ & $<0.01$ & 0.02 \\
\hline $18: 1$ trans- 11 & 24.0 & 105 & 23.0 & 34.5 & 7.33 & 0.001 & $<0.001$ & 0.001 \\
\hline $18: 1$ trans- 12 & 3.67 & $15 \cdot 3$ & 6.03 & 16.9 & 0.975 & 0.05 & $<0.001$ & 0.67 \\
\hline $18: 1$ trans $-13+14$ & 8.57 & 29.8 & $12 \cdot 8$ & $35 \cdot 2$ & 2.028 & 0.03 & $<0.001$ & 0.76 \\
\hline $18: 1$ trans -15 & $4 \cdot 31$ & 13.6 & $6 \cdot 13$ & $15 \cdot 4$ & 0.866 & 0.04 & $<0.001$ & 0.98 \\
\hline $18: 1$ trans- $16 \S$ & 4.60 & 14.9 & 6.54 & $15 \cdot 2$ & 1.051 & 0.22 & $<0.001$ & 0.36 \\
\hline \multicolumn{9}{|l|}{ Non-conjugated 18:2 (g/d) } \\
\hline $18: 2$ cis- 6, cis- 12 & 0.47 & 0.31 & 0.29 & 0.61 & 0.034 & 0.13 & 0.05 & $<0.001$ \\
\hline $18: 2$ cis- 7, cis- 12 & 0.39 & 0.42 & 0.29 & 0.73 & 0.045 & 0.06 & $<0.01$ & $<0.01$ \\
\hline $18: 2$ cis- 9, cis- 12 & 13.6 & $16 \cdot 6$ & 25.5 & $25 \cdot 6$ & $2 \cdot 18$ & $<0.001$ & 0.39 & 0.42 \\
\hline $18: 2$ cis-9, cis-15\| & 0.59 & 0.61 & 0.66 & 0.58 & 0.050 & 0.61 & 0.42 & 0.18 \\
\hline $18: 2$ cis- 12, cis- 15 & 0.35 & 0.27 & 0.24 & 0.16 & 0.039 & 0.01 & 0.04 & 0.90 \\
\hline $18: 2$ cis- 9, trans -12 & 0.25 & 0.25 & 0.25 & 0.43 & 0.020 & $<0.01$ & $<0.01$ & $<0.01$ \\
\hline $18: 2$ cis -9, trans -13 & 0.13 & 0.30 & 0.10 & 1.09 & 0.054 & $<0.001$ & $<0.001$ & $<0.001$ \\
\hline $18: 2$ trans -9, cis -12 & 0.29 & 0.53 & 0.31 & 0.74 & 0.039 & 0.02 & $<0.001$ & 0.05 \\
\hline $18: 2$ trans -10, cis -15 & 0.32 & 0.40 & 0.43 & $2 \cdot 31$ & 0.550 & 0.12 & 0.12 & 0.15 \\
\hline $18: 2$ trans -11, cis- 15 & 3.75 & 3.69 & 2.43 & $2 \cdot 13$ & 0.293 & 0.001 & 0.50 & 0.65 \\
\hline $18: 2$ trans- 12, cis-15 & 0.50 & 0.42 & 0.36 & 0.44 & 0.065 & 0.29 & 0.98 & 0.21 \\
\hline $18: 2$ trans -9, trans -12 & 0.06 & 0.12 & 0.06 & 0.68 & 0.093 & 0.02 & $<0.01$ & 0.02 \\
\hline $18: 2$ trans -11, trans -15 & 0.87 & 0.93 & 0.42 & 0.29 & 0.078 & $<0.001$ & 0.66 & 0.27 \\
\hline \multicolumn{9}{|l|}{ Conjugated 18:2(mg/d) } \\
\hline CLA cis-9, cis-11 & $15 \cdot 7$ & 23.7 & 37.4 & $25 \cdot 8$ & 14.67 & 0.45 & 0.90 & 0.53 \\
\hline CLA cis-10, cis-12 & 7.51 & $13 \cdot 2$ & 11.3 & 2.02 & 4.208 & 0.27 & 0.58 & 0.05 \\
\hline CLA cis- 11 , cis- 13 & 3.49 & 18.9 & 23.3 & $21 \cdot 1$ & 9.44 & 0.29 & 0.51 & 0.39 \\
\hline CLA cis-9, trans- 11 & 2609 & 7060 & 5189 & 4999 & $1305 \cdot 3$ & 0.77 & 0.04 & 0.03 \\
\hline CLA cis-11, trans-13 & 6.09 & 3.36 & $15 \cdot 0$ & 6.29 & 3.663 & 0.16 & 0.17 & 0.44 \\
\hline CLA cis-12, trans- 14 & $36 \cdot 3$ & 30.7 & 68.8 & 21.4 & 19.83 & 0.58 & 0.23 & 0.33 \\
\hline CLA trans-9, cis-11 & 35.0 & 220 & 46.5 & 1127 & 340.4 & 0.20 & 0.10 & 0.21 \\
\hline CLA trans-10, cis-12 & 163 & 225 & 603 & 641 & $115 \cdot 2$ & $<0.01$ & 0.64 & 0.91 \\
\hline CLA trans- 11 , cis-13 & 537 & 256 & 127 & 44.0 & 34.86 & $<0.001$ & $<0.01$ & 0.03 \\
\hline CLA trans-12, cis- 14 & 84.9 & 62.6 & 129 & $40 \cdot 1$ & 14.30 & 0.46 & $<0.01$ & 0.05 \\
\hline CLA trans -8, trans -10 & 44.7 & $66 \cdot 4$ & 37.7 & $96 \cdot 6$ & $6 \cdot 61$ & 0.11 & $<0.001$ & 0.03 \\
\hline CLA trans -9, trans -11 & 238 & 995 & 515 & 822 & 146.0 & 0.65 & $<0.01$ & 0.08 \\
\hline CLA trans-10, trans -12 & 85.4 & 372 & 202 & 534 & $50 \cdot 1$ & $<0.01$ & $<0.001$ & 0.55 \\
\hline CLA trans-11, trans -13 & 520 & 432 & 660 & 427 & 85.0 & 0.45 & 0.11 & 0.42 \\
\hline CLA trans-12, trans -14 & 186 & 138 & 105 & 121 & $20 \cdot 2$ & 0.04 & 0.44 & 0.15 \\
\hline CLA trans-13, trans-15 & $19 \cdot 2$ & $24 \cdot 1$ & $19 \cdot 1$ & 24.5 & 5.90 & 0.97 & 0.33 & 0.97 \\
\hline \multicolumn{9}{|l|}{$18: 3(\mathrm{~g} / \mathrm{d})$} \\
\hline $18: 3$ cis- 9, cis- 12, cis- 15 & 7.23 & 5.05 & 6.56 & 4.02 & 0.353 & 0.03 & $<0.001$ & 0.59 \\
\hline $18: 3$ cis- 9, trans- 11, trans -15 & 0.57 & 0.40 & 0.44 & 0.42 & 0.077 & 0.49 & 0.26 & 0.36 \\
\hline $18: 3$ cis- 9, trans -11, cis- 15 & 1.47 & 1.56 & 1.09 & 1.06 & 0.081 & $<0.01$ & 0.76 & 0.48 \\
\hline $\begin{array}{c}18: 3 \text { trans }-9, \text { trans- } 12, \text { cis- } 15+ \\
18: 3 \text { cis }-9, \text { cis- } 12, \text { trans }-15\end{array}$ & 0.13 & 0.09 & 0.08 & 0.07 & 0.013 & $<0.01$ & 0.03 & 0.23 \\
\hline $18: 3$ trans -9 trans -11 cis- 15 & 0.07 & 0.06 & 0.11 & 0.05 & 0.020 & 0.40 & 0.10 & 0.17 \\
\hline
\end{tabular}

L, low-concentrate diet (FC ratio 65:35 on a DM basis) with no additional lipid; LSO, low-concentrate diet (FC ratio 65:35) containing 50 g SO/kg diet DM; H, high-concentrate diet (FC ratio 35:65) with no additional lipid; HSO, high-concentrate diet (FC ratio 35:65) containing $50 \mathrm{~g} \mathrm{SO} / \mathrm{kg}$ diet DM.

* Values represent the mean over days 22-24 of each omasal digesta sampling.

† Significance of effects due to dietary FC ratio, supplements of $\mathrm{SO}$ and their interaction $(\mathrm{FC} \times \mathrm{SO})$.

¥ Coelutes with cis-8, cis-12-18:2 as a minor component.

$\S$ Coelutes with cis-14-18:1.

II Coelutes with cis-9-19:1. 
Table 6. Effect of dietary forage:concentrate (FC) ratio and sunflower oil (SO) on the flow of odd- and branched-chain and dicarboxylic fatty acids at the omasum in lactating cows*

(Least-square mean values with their pooled standard errors; $n$ 16)

\begin{tabular}{|c|c|c|c|c|c|c|c|c|}
\hline \multirow[b]{2}{*}{ Flow $(g / d)$} & \multicolumn{4}{|c|}{ Treatment } & \multirow[b]{2}{*}{ SEM } & \multicolumn{3}{|c|}{$P \dagger$} \\
\hline & $\mathrm{L}$ & LSO & $\mathrm{H}$ & HSO & & $\mathrm{FC}$ & so & $\mathrm{FC} \times \mathrm{SO}$ \\
\hline 3-phenyl-3:0 & $14 \cdot 3$ & $12 \cdot 3$ & $8 \cdot 41$ & 7.00 & 0.931 & $<0.001$ & $0 \cdot 12$ & 0.76 \\
\hline $11: 0$ & 0.08 & 0.07 & 0.08 & 0.07 & 0.007 & 0.74 & $0 \cdot 11$ & 0.98 \\
\hline 12:0 dicarboxylic acid & 0.90 & 0.80 & 1.02 & 0.44 & 0.198 & 0.46 & 0.07 & $0 \cdot 17$ \\
\hline $13: 0$ iso & 0.38 & 0.34 & 0.33 & 0.29 & 0.035 & 0.23 & 0.34 & 0.96 \\
\hline $13: 0$ anteiso & 0.13 & 0.15 & 0.12 & 0.06 & 0.017 & 0.02 & 0.28 & 0.06 \\
\hline $14: 0$ iso & 1.06 & 0.93 & 1.01 & 0.76 & 0.209 & 0.41 & $0 \cdot 18$ & 0.67 \\
\hline $15: 0$ & 6.81 & $7 \cdot 25$ & $5 \cdot 13$ & 3.53 & 0.568 & $<0.01$ & 0.35 & 0.12 \\
\hline $15: 0$ iso & $2 \cdot 64$ & $2 \cdot 34$ & $2 \cdot 38$ & 1.77 & 0.165 & 0.05 & 0.03 & 0.39 \\
\hline 15: 0 anteiso & $6 \cdot 60$ & $5 \cdot 63$ & 6.99 & $4 \cdot 84$ & 0.280 & 0.42 & $<0.001$ & 0.04 \\
\hline $15: 1$ trans -5 & 1.37 & 0.99 & 0.99 & 0.67 & 0.053 & $<0.001$ & $<0.001$ & 0.60 \\
\hline $16: 0$ iso & 1.95 & 1.81 & 1.87 & 1.55 & 0.434 & $0 \cdot 70$ & 0.62 & 0.85 \\
\hline $17: 0$ & 3.29 & 3.51 & $3 \cdot 16$ & $3 \cdot 10$ & 0.273 & 0.36 & 0.80 & 0.62 \\
\hline $17: 0$ iso & 1.39 & 1.53 & 1.40 & 1.08 & 0.129 & 0.14 & 0.50 & $0 \cdot 12$ \\
\hline 17: 0 anteiso & 1.82 & 1.40 & 1.59 & 1.40 & 0.402 & 0.79 & 0.49 & 0.78 \\
\hline 11-cyclohexyl-11:0 & 1.90 & 1.65 & 3.17 & 3.21 & 0.434 & $<0.01$ & 0.78 & 0.71 \\
\hline $17: 1$ trans- 10 & 0.23 & 0.21 & 0.19 & 0.18 & 0.017 & 0.05 & 0.31 & 0.92 \\
\hline $18: 0$ iso & 0.36 & 0.30 & 0.28 & 0.17 & 0.027 & $<0.01$ & 0.02 & 0.43 \\
\hline $19: 0$ & 0.34 & 0.49 & 0.39 & 0.55 & 0.030 & $0 \cdot 10$ & $<0.01$ & 0.93 \\
\hline S3,R7,R11,15-tetramethyl-16:0 & 4.75 & $7 \cdot 64$ & 4.03 & 0.15 & 0.501 & $<0.001$ & 0.36 & $<0.001$ \\
\hline R3,R7,R11,15-tetramethyl-16:0 & 1.43 & 0.29 & 0.28 & 0.10 & 0.107 & $<0.001$ & $<0.001$ & $<0.01$ \\
\hline $21: 0$ & 0.25 & 0.27 & 0.23 & 0.22 & 0.018 & 0.16 & 0.85 & 0.41 \\
\hline $23: 0$ & 0.67 & 0.90 & 0.66 & 0.82 & 0.048 & 0.39 & $<0.01$ & 0.53 \\
\hline $23: 1$ cis-14 & 0.19 & 0.21 & 0.21 & 0.18 & 0.018 & 0.60 & 0.53 & 0.03 \\
\hline $25: 0$ & 0.35 & 0.42 & 0.32 & 0.29 & 0.024 & 0.02 & 0.42 & 0.09 \\
\hline $25: 1$ cis-16 & 0.11 & $0 \cdot 11$ & 0.12 & $0 \cdot 10$ & 0.011 & 0.68 & 0.37 & 0.42 \\
\hline $27: 0$ & 0.18 & 0.23 & 0.12 & 0.08 & 0.009 & $<0.001$ & 0.09 & $<0.001$ \\
\hline $29: 0$ & 0.15 & 0.22 & 0.11 & 0.06 & 0.022 & $<0.01$ & 0.67 & 0.03 \\
\hline
\end{tabular}

L, low-concentrate diet (FC ratio 65:35 on a DM basis) with no additional lipid; LSO, low-concentrate diet (FC ratio 65:35) containing 50 g SO/kg diet DM; $\mathrm{H}$, high-concentrate diet (FC ratio 35:65) with no additional lipid; HSO, high-concentrate diet (FC ratio 35:65) containing $50 \mathrm{~g} \mathrm{SO} / \mathrm{kg}$ diet DM.

* Values represent the mean over days 22-24 of each omasal digesta sampling.

$\dagger$ Significance of effects due to dietary FC ratio, supplements of $\mathrm{SO}$ and their interaction $(\mathrm{FC} \times \mathrm{SO})$

resulted in a greater increase of trans-12-20:1 at the omasum compared with the $\mathrm{L}$ diet $(P=0.05$ for $\mathrm{FC} \times \mathrm{SO}$ interaction).

Dietary FC ratio and SO supplements altered the flow of odd- and branched-chain fatty acids (OBCFA) at the omasum (Table 6). Flows of 3-phenyl-3: 0, anteiso-13:0, 15:0, iso-15:0, trans-5-15:1, trans-10-17:1, iso-18:0 and 25:0 were decreased $(P \leq 0.05)$ and that of 11-cyclohexyl-11:0 was increased $(P<0 \cdot 01)$ on $\mathrm{H}$ compared with $\mathrm{L}$ treatments. SO increased $(P<0.01) 19: 0$ and $23: 0$, but lowered $(P<0.05)$ iso-15:0, trans-5-15:1 and iso-18:0 at the omasum. Flows of S3,R7,R11,15-tetramethyl-16:0, cis-14-23:1, $27: 0$ and 29:0 were increased by SO supplements in the $\mathrm{L}$ diet, but decreased when included in the $\mathrm{H}$ diet $(P<0.05$ for $\mathrm{FC} \times \mathrm{SO}$ interaction). Furthermore, decreases in the amounts of R3,R7,R11,15-tetramethyl-16:0 at the omasum were greater when SO was included in the $\mathrm{L}$ than $\mathrm{H}$ diet, whereas the reverse was true for anteiso-15: $0(P<0.05$ for $\mathrm{FC} \times \mathrm{SO}$ interaction $)$.

SO lowered $(P<0 \cdot 01)$ total DMA flow at the omasum, decreases that tended to be greater when included in the $\mathrm{H}$ than $\mathrm{L}$ diet $(P=0.06$ for $\mathrm{FC} \times \mathrm{SO}$ interaction; Table 7$)$. Furthermore, $\mathrm{SO}$ decreased $(P<0.05)$ DMA of $15: 0,16: 0,17: 0$, iso-17:0 and $18: 0$ at the omasum. Increases in dietary concentrates lowered $(P<0.05)$ DMA of 13:0, iso-14:0, 15:0, anteiso-15:0, iso-15:0, $26: 0,28: 0$ and $30: 0$, and increased $(P<0.05)$ DMA of $16: 0$, $17: 0$, iso-17:0, trans-10-18:1 and cis-9-18:1. Inclusion of SO resulted in larger decreases in DMA of trans-11-18: 1, cis-11-18: 1 and cis-12-18: 1 at the omasum when included in the $\mathrm{H}$ than $\mathrm{L}$ diet $(P<0.05$ for $\mathrm{FC} \times \mathrm{SO}$ interaction), whereas the decrease in anteiso-13: 0 DMA to SO was higher when included in the $\mathrm{L}$ than $\mathrm{H}$ diet $(P<0.05$ for $\mathrm{FC} \times \mathrm{SO}$ interaction $)$.

\section{Ruminal biohydrogenation}

Dietary FC ratio had no effect $(P>0.05)$ on apparent biohydrogenation of 18-C unsaturated FA whilst SO increased $(P<0 \cdot 001)$ apparent biohydrogenation of cis-9-18:1, 18:2n-6 and $18: 3 n-3$ in the rumen (Table 8 ). No 18: $4 n-3$ was detected in omasal digesta.

\section{Discussion}

High-concentrate diets containing high amounts of PUFA typically cause MFD in lactating cows ${ }^{(9-11)}$. Changes in milk fat composition and secretion during diet-induced MFD on low fibre high-oil diets have been characterised ${ }^{(4-7)}$ but quantitative measurements of ruminal lipid metabolism and flow of FA escaping the rumen are limited ${ }^{(17,25,28)}$. Measurements of FA and DMA at the omasum were performed in cows fed diets formulated to cause changes in ruminal lipid metabolism and milk fat synthesis allowing alterations in ruminal biohydrogenation and formation of intermediates associated with a diet causing MFD to be characterised in detail. 
Table 7. Effect of dietary forage:concentrate $(\mathrm{FC})$ ratio and sunflower oil (SO) on the flow of aldehydes at the omasum in lactating cows ${ }^{*}$ (Least-square mean values with their pooled standard errors; $n$ 16)

\begin{tabular}{|c|c|c|c|c|c|c|c|c|}
\hline \multirow[b]{2}{*}{ Flow $(g / d)$} & \multicolumn{4}{|c|}{ Treatment } & \multirow[b]{2}{*}{ SEM } & \multicolumn{3}{|c|}{$P \dagger$} \\
\hline & $\mathrm{L}$ & LSO & $\mathrm{H}$ & HSO & & $\mathrm{FC}$ & so & $\mathrm{FC} \times \mathrm{SO}$ \\
\hline DMA-12:0 & 0.60 & 0.46 & 0.45 & 0.39 & 0.057 & 0.09 & 0.10 & 0.45 \\
\hline DMA-13:0 & 0.31 & 0.35 & 0.22 & $0 \cdot 10$ & 0.045 & $<0.01$ & 0.35 & 0.12 \\
\hline DMA-13:0 anteiso & 0.27 & 0.18 & 0.20 & 0.19 & 0.015 & 0.12 & 0.02 & 0.05 \\
\hline DMA-13:0 iso & 0.08 & 0.07 & 0.09 & 0.09 & 0.019 & 0.53 & 0.96 & 0.71 \\
\hline DMA-14:0 & 1.54 & 1.45 & 1.77 & 1.09 & 0.193 & 0.76 & 0.09 & 0.18 \\
\hline DMA-14:0 iso & 1.02 & 0.98 & 0.86 & 0.62 & 0.132 & 0.04 & 0.23 & 0.34 \\
\hline DMA-15:0 & 1.79 & 1.46 & 1.51 & 0.90 & 0.108 & $<0.01$ & $<0.01$ & 0.20 \\
\hline DMA-15:0 anteiso & 2.42 & $2 \cdot 32$ & $2 \cdot 19$ & 1.24 & 0.223 & 0.03 & 0.06 & 0.11 \\
\hline DMA-15: 0 iso & $1 \cdot 18$ & $1 \cdot 18$ & 0.90 & 0.59 & 0.101 & $<0.01$ & 0.17 & 0.18 \\
\hline DMA-16:0 & 6.02 & $5 \cdot 62$ & $10 \cdot 1$ & $7 \cdot 14$ & 0.659 & $<0.01$ & 0.04 & $0 \cdot 10$ \\
\hline DMA-16:0 iso & 0.63 & 0.56 & 1.01 & 0.89 & 0.232 & 0.18 & 0.70 & 0.92 \\
\hline DMA-17:0 & 0.10 & 0.07 & 0.13 & 0.08 & 0.006 & 0.03 & $<0.001$ & 0.11 \\
\hline DMA-17:0 anteiso & 0.16 & 0.11 & 0.22 & 0.31 & 0.121 & 0.34 & 0.86 & 0.58 \\
\hline DMA-17:0 iso & 0.10 & 0.08 & 0.19 & 0.09 & 0.016 & 0.02 & $<0.01$ & 0.06 \\
\hline DMA-18:0 & 0.86 & 0.56 & 1.12 & 0.50 & 0.083 & 0.25 & 0.001 & $0 \cdot 10$ \\
\hline DMA-18: 1 trans-10 & $<0.01$ & $<0.01$ & 0.27 & 0.33 & 0.082 & 0.01 & 0.70 & 0.70 \\
\hline DMA-18: 1 trans- 11 & 0.27 & 0.21 & 0.55 & 0.19 & 0.056 & 0.06 & $<0.01$ & 0.04 \\
\hline DMA-18: 1 cis-9 & 1.01 & 0.79 & 2.33 & 1.27 & 0.302 & 0.02 & 0.08 & 0.21 \\
\hline DMA-18: 1 cis-11 & 0.48 & 0.35 & 1.07 & 0.58 & 0.062 & $<0.001$ & $<0.01$ & 0.03 \\
\hline DMA-18: 1 cis-12 & 0.26 & 0.27 & 0.59 & 0.24 & 0.047 & $<0.01$ & $<0.01$ & $<0.01$ \\
\hline DMA-26:0 & 0.66 & 0.53 & 0.44 & 0.33 & 0.051 & $<0.01$ & 0.06 & 0.83 \\
\hline DMA-28:0‡ & 1.00 & 0.84 & 0.73 & 0.59 & 0.088 & 0.02 & 0.12 & 0.92 \\
\hline DMA-30:0 & 0.59 & 0.50 & 0.43 & 0.34 & 0.050 & 0.02 & 0.11 & 0.99 \\
\hline$\sum$ DMA & 21.4 & 18.9 & $27 \cdot 4$ & $18 \cdot 1$ & 1.47 & 0.13 & $<0.01$ & 0.06 \\
\hline
\end{tabular}

$\mathrm{L}$, low-concentrate diet (FC ratio 65:35 on a DM basis) with no additional lipid; LSO, low-concentrate diet (FC ratio 65:35) containing 50 g SO/kg diet DM; $\mathrm{H}$, high-concentrate diet (FC ratio 35:65) with no additional lipid; HSO, high-concentrate diet (FC ratio 35:65) containing $50 \mathrm{~g} \mathrm{SO} / \mathrm{kg}$ diet DM; DMA, dimethyl acetal.

* Values represent the mean over days 22-24 of each omasal digesta sampling.

$\dagger$ Significance of effects due to dietary FC ratio, supplements of $\mathrm{SO}$ and their interaction $(\mathrm{FC} \times \mathrm{SO})$.

$\ddagger$ Coelutes with 9-oxo-18:0.

Table 8. Effect of dietary forage:concentrate (FC) ratio and sunflower oil (SO) on apparent biohydrogenation of 18-carbon unsaturated fatty acids in the rumen of lactating cows*

(Least-square mean values with their pooled standard errors; $n$ 16)

\begin{tabular}{|c|c|c|c|c|c|c|c|c|}
\hline \multirow[b]{2}{*}{ Biohydrogenation (\%) } & \multicolumn{4}{|c|}{ Treatment } & \multirow[b]{2}{*}{ SEM } & \multicolumn{3}{|c|}{$P+$} \\
\hline & L & LSO & $\mathrm{H}$ & $\mathrm{HSO}$ & & $\mathrm{FC}$ & so & $\mathrm{FC} \times \mathrm{SO}$ \\
\hline $18: 1$ cis-9 & 70.7 & 89.8 & $67 \cdot 2$ & $91 \cdot 1$ & 3.03 & 0.71 & $<0.001$ & 0.42 \\
\hline $18: 1$ cis- 11 & $50 \cdot 8$ & $62 \cdot 7$ & 31.3 & 52.0 & 0.72 & 0.08 & 0.06 & 0.56 \\
\hline $18: 2$ cis- 9, cis -12 & $85 \cdot 1$ & $97 \cdot 1$ & $84 \cdot 3$ & $96 \cdot 3$ & $1 \cdot 32$ & 0.54 & $<0.001$ & 0.99 \\
\hline $18: 3$ cis -9, cis- 12, cis- 15 & $91 \cdot 6$ & $94 \cdot 1$ & $90 \cdot 8$ & 93.7 & 0.40 & $0 \cdot 19$ & $<0.001$ & 0.61 \\
\hline
\end{tabular}

$\mathrm{L}$, low-concentrate diet (FC ratio 65:35 on a DM basis) with no additional lipid; LSO, low-concentrate diet (FC ratio 65:35) containing 50 g SO/kg diet DM; H, high-concentrate diet (FC ratio 35:65) with no additional lipid; HSO, high-concentrate diet (FC ratio 35:65) containing $50 \mathrm{~g} \mathrm{SO} / \mathrm{kg}$ diet DM.

* Values represent the mean over days $22-24$ of each omasal digesta sampling.

† Significance of effects due to dietary FC ratio, supplements of SO and their interaction $(\mathrm{FC} \times \mathrm{SO})$.

By design, inclusion of SO in the high-concentrate diet decreased milk fat content and secretion relative to other treatments (mean decreases of -25.4 to -30.3 and -20.2 to $-31.9 \%$, respectively). The magnitude of decreases in milk fat synthesis on the HSO diet is within the expected range reported for cows fed low fibre high-oil diets ${ }^{(9,10)}$ or receiving post-ruminal infusions of FA preparations containing trans-10, cis-12-CLA $^{\text {(29) }}$ and therefore representative of the MFD phenotype. In more extreme cases, decreases in milk fat on diets causing MFD can approach a physiological maximum of $-50 \%$, but this is often accompanied by lowered intake ${ }^{(6,7,30)}$ complicating the establishment of cause and effect.

Flow of total FA at the omasum was higher than intake on all treatments $(344,321,353$ and $216 \mathrm{~g} / \mathrm{d}$ for L, LSO, H and HSO, respectively). The higher FA flow can be explained by the contribution of microbial lipid to total FA escaping the rumen. On most diets, dietary intake accounts for between 75 and $80 \%$ of FA reaching the duodenum ${ }^{(31,32)}$. Dietary lipid supplements containing PUFA have been shown to lower the net balance of FA in the rumen of lactating and growing cattle ${ }^{(31,32)}$, but there were no indications in the present study that SO, even when offered in relatively high amounts, decreased FA flow at the omasum relative to intake.

On all diets, the intake of cis-9-18:1, cis-11-18:1, 18:2n-6 and $18: 3 n-3$ exceeded flow at the omasum. Dietary FC ratio did not alter the extent of FA biohydrogenation in the rumen, whereas SO increased apparent ruminal biohydrogenation of unsaturated FA. Increases in dietary concentrates have been 
reported to have no effect on the extent of cis-9-18:1 metabolism, but decrease ruminal biohydrogenation of $18: 2 n-6$ and $18: 3 n-3$ in lactating cows ${ }^{(17,33,34)}$. More extensive biohydrogenation of unsaturated FA on diets containing SO was associated with higher dietary FA intake consistent with the findings of a recent meta-analysis examining the relationship between the intake and flow of FA at the duodenum in ruminants ${ }^{(35)}$. These observations provide further support that the main role of biohydrogenation in the rumen is to decrease the abundance of unsaturated FA in the rumen to minimise the inhibitory effects on microbial growth.

Although SO resulted in a higher 18:2n-6 intake, the amount reaching the omasum was not increased. Lipolysis is considered rate limiting for the conversion of unsaturated FA to saturated end products in the rumen ${ }^{(36)}$. During incubations with rumen contents, lipolysis of SO is decreased when added at high concentrations $^{(37)}$ or increased when fibre replaces $\operatorname{starch}^{(38)}$. Lipolysis was not determined, but substantial metabolism of 18:2n-6 between the mouth and omasum on LSO and HSO treatments do not support hydrolysis of ester bonds of TAG in SO limiting biohydrogenation of unsaturated FA or that diet composition has a substantial influence on ruminal lipolysis of SO in vivo. Flow of $18: 2 n-6$ at the omasum was almost 2 -fold greater on $\mathrm{H}$ than $\mathrm{L}$ diets that is in agreement with earlier reports of higher ruminal escape of $18: 2 n-6$ in lactating cows fed high-concentrate diets ${ }^{(17,33,34)}$. It has been suggested that non-esterified $18: 2 n-6$ could be selectively incorporated into the vacuoles of rumen bacteria ${ }^{(39)}$, minimising exposure to bacterial isomerases and reductases in the rumen. However, direct measurement of flows of $18: 2 n-6$ at the omasum relative to intake do not suggest this is quantitatively important.

On all treatments, the reduction of dietary unsaturated FA in the rumen was incomplete and numerous $16: 1,18: 1,18: 2$ and $20: 1$ intermediates not supplied from the diet were detected in omasal digesta. Even though the extent of unsaturated FA biohydrogenation in the rumen did not differ between oil containing diets inclusion of $\mathrm{SO}$ in the high-concentrate diet altered ruminal biohydrogenation pathways.

During incubations with strained rumen contents $18: 2 n-6$ is rapidly isomerised to yield geometric isomers of $\Delta 9,11$ and $\Delta 10,12-$ CLA $^{(12,40,41)}$. The amount of trans-10, cis-12-CLA at the omasum was higher on $\mathrm{H}$ than $\mathrm{L}$ diets but not altered in response to SO. Earlier experiments have shown that ruminal escape of trans-10, cis-12-CLA is promoted on low-fibre $\operatorname{diets}^{(17,31)}$ and in response to increased $18: 2 n-6$ intake $^{(42,43)}$. Under the specified conditions of this experiment, MFD on the HSO treatment was not accompanied by an increase in trans-10, cis-12-CLA that is known to inhibit milk fat synthesis in lactating cows ${ }^{(9,13,14)}$

Incubations with mixed rumen contents or pure strains of rumen bacteria ${ }^{(12,40,41)}$ have shown that trans-10, trans-12-CLA is formed from $18: 2 n-6$. Flows of trans-10, trans-12-CLA at the omasum were increased by $\mathrm{SO}$ and also higher on $\mathrm{H}$ than $\mathrm{L}$ diets suggesting that substrate supply and rumen environment may influence the specificity of the $\Delta-12$ cis, $\Delta-11$ trans isomerase or the rate at which 10,12-CLA products are reduced. However, flow of trans-10, trans-12-CLA at the omasum was not increased on the HSO treatment causing MFD. Post-ruminal infusion of geometric 10,12 CLA isomers has implicated cis-10, trans-12-CLA as a possible milk fat inhibitor ${ }^{(15)}$, but no cis-10, trans-12-CLA was detected in omasal digesta consistent with earlier reports ${ }^{(27,28,42)}$

Flow of cis-9, trans-11-CLA was increased during SO supplementation of the $\mathrm{L}$ but not $\mathrm{H}$ diet consistent with reports in growing cattle that sources of $18: 2 n-6$ promote cis-9, trans-11-CLA at the duodenum on high-forage diets ${ }^{(43)}$. In contrast, the flow of cis-9, trans-11-CLA at the duodenum was found to be higher in cows following the inclusion of linseed oil into a high- rather than low-concentrate diet ${ }^{(17)}$. Such findings indicate that substrate supply is not the sole factor regulating the synthesis of cis-9, trans-11-CLA in the rumen. Even though cis-9, trans-11-CLA is the main product formed during the isomerisation of $18: 2 n-6$ in the rumen ${ }^{(44)}$, other geometric 9,11 CLA isomers are also formed $^{(12,40,41)}$. On both $\mathrm{L}$ and $\mathrm{H}$ diets, SO increased trans-9, cis-11-CLA at the omasum, with the amounts on the highconcentrate diet being numerically several-fold higher, consistent with the observed increases in milk trans-9, cis-11-CLA concentrations on low fibre high-oil diets causing MFD ${ }^{(6,16)}$. Abomasal infusion of a mixture of FA has identified trans-9, cis-11-CLA as a possible inhibitor of milk fat synthesis in the lactating cow ${ }^{(16)}$.

Irrespective of dietary lipid content, trans-11-18:1 was the major biohydrogenation intermediate on the $\mathrm{L}$ treatments. However, including $\mathrm{SO}$ in the $\mathrm{H}$ diet caused a shift in biohydrogenation pathways resulting in the formation of trans-10 containing products, with the most obvious being trans-10-18:1 replacing trans-11-18:1 as the major biohydrogenation intermediate formed in the rumen. Alterations in ruminal biohydrogenation favouring the synthesis of trans-10$18: 1$ are known to occur in lactating cows fed high-concentrate $\operatorname{diets}^{(28)}$, high-starch low-fibre diets containing plant oils ${ }^{(17)}$ or diets supplemented with high amounts of PUFA ${ }^{(25)}$. However, the amount of trans-10-18:1 formed on the HSO treatment $(133 \mathrm{~g} / \mathrm{d})$ is much higher compared with earlier reports in lactating cows fed diets causing MFD ${ }^{(10,25,28)}$. Changes in diet composition promoting the formation of trans-10-18:1 are well characterised $^{(9,10)}$, but the underlying causes are not known. Examination of the effects of $\mathrm{pH}$ or dietary $\mathrm{FC}$ ratio on the production of biohydrogenation intermediates in dual-flow continuous culture suggested that decreases in $\mathrm{pH}$ rather than increases in the proportion of concentrates is the main factor responsible for the shift from trans-11-18:1 to trans-10$18: 1^{(45)}$. In contrast, measurements of ruminal FA composition in cows fed diets containing low or high amounts of starch ${ }^{(46)}$ or FA flow at the omasum in cows fed incremental amounts of fish oil ${ }^{(25)}$ indicate that trans-10-18:1 formation in vivo to be independent of decreases in rumen $\mathrm{pH}$. In the present experiment, greater formation of trans-10-18: 1 on the HSO treatment was not associated with a decrease in mean daily rumen $\mathrm{pH}$ (results not presented). Trans-10-18:1 is thought to originate from the reduction of trans-10, cis-12-CLA ${ }^{(47)}$. Therefore, a close association between trans-10-18:1 and trans-10, cis-12-CLA at the omasum might be expected, but none was observed. Concentrations of trans-10-18:1 are known to increase in milk from cows fed diets causing $\operatorname{MFD}^{(9,10)}$, but direct evidence that trans-10-18:1 inhibits milk fat synthesis remains equivocal. Abomasal infusion of trans-10-18:1 was reported to have no 
effect on milk fat secretion ${ }^{(48)}$, but these findings have been challenged on the basis that the effective dose at the mammary gland was too low for possible inhibitory effects to be detected $^{(49)}$. A more recent experiment demonstrated that post-ruminal infusion of a mixture of 18:1 methyl esters containing trans-10-18:1 lowered milk fat yield in lactating cows ${ }^{(50)}$. Further investigations are required to confirm the role of trans-10-18:1 in the regulation of milk fat synthesis.

Ruminal biohydrogenation of $18: 3 n-3$ involves an initial isomerisation of the cis-12 double bond to yield cis-9, trans-11, cis-15-18: 3 that is reduced to trans-11, cis-15-18: $2^{(36)}$. A higher intake of $18: 3 n-3$ can account for the increases in cis-9, trans11, cis-15-18:3, trans-11, cis-15-18: 2 and trans-11, trans-15$18: 2$ at the omasum on $\mathrm{L}$ than $\mathrm{H}$ treatments. Even though SO did not increase $18: 3 n-3$ intake the amount of trans-10, cis-15$18: 2$ at the omasum was higher for HSO than LSO providing additional support for alternative pathways of $18: 3 n-3$ metabolism in the rumen ${ }^{(51)}$ that appear quantitatively more important in cows fed diets causing MFD.

Appearance of OBCFA at the omasum originate from bacterial membrane lipids synthesised in the rumen using branched-chain amino acids as a primer ${ }^{(52)}$, with the relative abundances of OBCFA differing between bacterial species ${ }^{(53)}$. Increases in dietary concentrate decreased the flow of several (3-phenyl-3:0, anteiso-13:0, 15:0, iso- $15: 0$, trans $-5-15: 1$, trans $-10-17: 1$, iso- $18: 0$ and $25: 0$ ) OBCFA, consistent with earlier studies ${ }^{(17)}$. Such changes may reflect both changes in the rumen microbial community structure and the availability of substrates for bacterial OBCFA synthesis de novo. Total OBCFA at the omasum was lowest on the HSO diet, that is in agreement with earlier reports that lipid supplements decrease the proportion of OBCFA in lipid at the duodenum in cattle ${ }^{(17,54)}$, possibly due to inhibition of bacterial FA synthesis or by dilution with dietary lipid escaping the rumen. Membrane lipid of rumen bacteria is also characterised by the occurrence of DMA, but little is known on dietary factors influencing DMA synthesis in the rumen. SO tended to lower total DMA at the omasum, whereas the relative proportions of concentrate and forage in the diet had a larger influence, findings that are in agreement with recent reports on the effect of diet on DMA in rumen contents of sheep ${ }^{(26)}$.

\section{Conclusions}

Diets containing different proportions of concentrate and SO supplements resulted in varying effects on ruminal lipid metabolism and milk fat synthesis. Inclusion of SO in a high-concentrate diet caused MFD that was accompanied by alterations in ruminal biohydrogenation pathways promoting the formation of trans-10 containing intermediates. However, ruminal synthesis of trans-10, cis-12-CLA known to inhibit milk fat synthesis was not increased on a high-concentrate diet containing SO, suggesting that ruminal formation of other biohydrogenation intermediates or additional mechanisms also contribute to the MFD phenotype in lactating cows.

\section{Acknowledgements}

The authors gratefully acknowledge the contribution of staff at the Metabolism Unit of Natural Resources Institute Finland
(Luke; Jokioinen, Finland) for the care of experimental animals and the collection of experimental samples under the supervision of Mari Talvisilta, and fatty acid analysis undertaken in the Luke laboratory by Minna Aalto and Tuija Hakala.

This work was supported by the Finnish Ministry of Agriculture and Forestry, Helsinki, Finland and Valio, Ltd, Helsinki, Finland. L. V. was a recipient of a PhD studentship from the Raisio Science Foundation, Raisio, Finland and the August Johannes and Aino Tiura Agricultural Science Foundation, Helsinki, Finland. None of the funding agencies had role in experimental design, data analysis or writing of this article.

The authors' contributions are as follows: K. J. S. and J. V. designed the experiment; L. V., P. K., H. L. and T. S. performed the research; L. V., H. L., A. R. B. and K. J. S. analysed experimental data; K. J. S. and L. V. wrote the paper; and K. J. S. had primary responsibility for the final content. All authors have read and approved the final manuscript contents.

The authors have no financial or personal conflicts of interest to declare.

\section{Supplementary material}

For supplementary material/s referred to in this article, please visit https://doi.org/10.1017/S0007114517000010

\section{References}

1. World Health Organization/Food Agricultural Organization (2003) Diet, nutrition and the prevention of chronic diseases. Report of a joint WHO/FAO expert consultation. World Health Organization Technical Report Series 916, 149 pp. http://www.who.int/dietphysicalactivity/publications/ trs916/download/en/ (accessed September 2015).

2. Hulshof KFAM, van Erp-Baart MA, Anttolainen M, et al. (1999) Intake of fatty acids in Western Europe with emphasis on trans fatty acids: the TRANSFAIR study. Eur J Clin Nutr $\mathbf{5 3}$, 143-157.

3. Shingfield KJ, Bonnet M \& Scollan ND (2013) Recent developments in altering the fatty acid composition of ruminantderived foods. Animal 7, Suppl. 1, 132-162.

4. Griinari JM, Dwyer DA, McGuire MA, et al. (1998) Trans-octadecenoic acids and milk fat depression in lactating dairy cows. J Dairy Sci 81, 1251-1261.

5. Peterson DG, Matitashvili EA \& Bauman DE (2003) Diet-induced milk fat depression in dairy cows results in increased trans-10, cis-12 CLA in milk fat and coordinate suppression of mRNA abundance for mammary enzymes involved in milk fat synthesis. J Nutr 133, 3098-3102.

6. Roy A, Ferlay A, Shingfield KJ, et al. (2006) Examination of the persistency of milk fatty acid composition responses to plant oils in cows given different basal diets, with particular emphasis on trans-C18:1 fatty acids and isomers of conjugated linoleic acid. Anim Sci 82, 479-492.

7. Rico DE \& Harvatine KJ (2013) Induction of and recovery from milk fat depression occurs progressively in dairy cows switched between diets that differ in fiber and oil concentration. J Dairy Sci 96, 6621-6630.

8. Bauman DE \& Griinari JM (2001) Regulation and nutritional manipulation of milk fat: low-fat milk syndrome. Livest Prod Sci 70, 15-29.

9. Bauman DE \& Griinari JM (2003) Nutritional regulation of milk fat synthesis. Annu Rev Nutr 23, 203-227. 
10. Shingfield KJ \& Griinari JM (2007) Role of biohydrogenation intermediates in milk fat depression. Eur J Lipid Sci Technol 109, 799-816.

11. Harvatine KJ, Boisclair YR \& Bauman DE (2009) Recent advances in the regulation of milk fat synthesis. Animal 3, 40-54.

12. Wallace RJ, McKain N, Shingfield KJ, et al. (2007) Isomers of conjugated linoleic acids are synthesized via different mechanisms in ruminal digesta and bacteria. J Lipid Res 48, 2247-2254.

13. Baumgard LH, Corl BA, Dwyer DA, et al. (2000) Identification of the conjugated linoleic acid isomer that inhibits milk fat synthesis. Am J Physiol Regul Integr Comp Physiol 278, R179-R184.

14. Glasser F, Ferlay A, Doreau M, et al. (2010) t10,c12-18: 2 -Induced milk fat depression is less pronounced in cows fed high-concentrate diets. Lipids 45, 877-887.

15. Sæbø A, Sæbø P-C, Griinari JM, et al. (2005) Effect of abomasal infusions of geometric isomers of 10,12 conjugated linoleic acid on milk fat synthesis in dairy cows. Lipids $\mathbf{4 0}, 823-832$.

16. Perfield JW II, Lock AL, Griinari JM, et al. (2007) Trans-9, cis-11 conjugated linoleic acid reduces milk fat synthesis in lactating dairy cows. J Dairy Sci 90, 2211-2218.

17. Loor JJ, Ueda K, Ferlay A, et al. (2004) Biohydrogenation, duodenal flow, and intestinal digestibility of trans fatty acids and conjugated linoleic acids in response to dietary forage concentrate ratio and linseed oil in dairy cows. J Dairy Sci 87, 2472-2485.

18. European Council (1986) Council Directive of 24 November on the approximation of laws, regulations and administrative provisions of the Member States regarding the protection of animals used for experimental and other scientific purposes (86/609/EEC). Official Journal of the European Communities L358, 1-28.

19. Shingfield KJ, Jaakkola S \& Huhtanen P (2001) Effects of level of nitrogen fertilizer application and various nitrogenous supplements on milk production and nitrogen utilization of dairy cows fed grass silage-based diets. Anim Sci 73, 541-554.

20. Ahvenjärvi S, Vanhatalo A, Huhtanen P, et al. (2000) Determination of reticulo-rumen and whole-stomach digestion in lactating cows by omasal canal or duodenal sampling. Br J Nutr 83, 67-77.

21. Shingfield KJ, Ärölä A, Ahvenjärvi S, et al. (2008) Ruminal infusions of cobalt-EDTA reduce mammary $\Delta^{9}$-desaturase index and alter milk fatty acid composition in lactating cows. J Nutr 138, 710-717.

22. Binnerts WT, van't Klooster AT \& Frens AM (1968) Soluble chromium indicator measured by atomic absorption in digestion experiments. Vet Rec 82, 470.

23. Shingfield KJ, Ahvenjärvi S, Toivonen V, et al. (2003) Effect of dietary fish oil on biohydrogenation of fatty acids and milk fatty acid content in cows. Anim Sci 77, 165-179.

24. Halmemies-Beauchet-Filleau A, Kairenius $P$, Ahvenjärvi S, et al. (2013) Effect of forage conservation method on ruminal lipid metabolism and microbial ecology in lactating cows fed diets containing a 60:40 forage-to-concentrate ratio. J Dairy Sci 96, 2428-2447.

25. Shingfield KJ, Kairenius P, Ärölä A, et al. (2012) Dietary fish oil supplements modify ruminal biohydrogenation, alter the flow of fatty acids at the omasum, and induce changes in the ruminal Butyrivibrio population in lactating cows. $J$ Nutr 142, 1437-1448.

26. Alves SP, Santos-Silva J, Cabrita ARJ, et al. (2013) Detailed dimethylacetal and fatty acid composition of rumen content from lambs fed lucerne or concentrate supplemented with soybean oil. PLOS ONE 8, e58386.

27. Stroup WW (2012) Generalized Linear Mixed Models: Modern Concepts, Methods and Applications. Boca Raton, FL: CRC Press.
28. Piperova LS, Sampugna J, Teter BB, et al. (2002) Duodenal and milk trans octadecenoic acid and conjugated linoleic acid (CLA) isomers indicate that postabsorptive synthesis is the predominant source of cis-9-containing CLA in lactating dairy cows. J Nutr 132, 1235-1241.

29. Harvatine KJ, Perfield JW II \& Bauman DE (2009) Expression of enzymes and key regulators of lipid synthesis is upregulated in adipose tissue during CLA-induced milk fat depression in dairy cows. J Nutr 139, 849-854.

30. Shingfield KJ, Reynolds CK, Hervás G, et al. (2006) Examination of the persistency of milk fatty acid composition responses to fish oil and sunflower oil in the diet of dairy cows. J Dairy Sci 89, 714-732.

31. Doreau M \& Ferlay A (1994) Digestion and utilisation of fatty acids by ruminants. Anim Feed Sci Technol 45, 379-396.

32. Schmidely P, Glasser F, Doreau M, et al. (2008) Digestion of fatty acids in ruminants: a meta-analysis of flows and variation factors. 1. Total fatty acids. Animal 2, 677-690.

33. Kalscheur KF, Teter BB, Piperova LS, et al. (1997) Effect of dietary forage concentration and buffer addition on duodenal flow of trans-C18:1 fatty acids and milk fat production in dairy cows. J Dairy Sci $\mathbf{8 0}, 2104-2114$.

34. Dewhurst RJ, Moorby JM, Vlaeminck B, et al. (2007) Apparent recovery of duodenal odd- and branched-chain fatty acids in milk of dairy cows. J Dairy Sci $\mathbf{9 0}, 1775-1780$.

35. Glasser F, Schmidely P, Sauvant D, et al. (2008) Digestion of fatty acids in ruminants: a meta-analysis of flows and variation factors: 2. C18 fatty acids. Animal 2, 691-704.

36. Jenkins TC, Wallace RJ, Moate PJ, et al. (2008) Board-invited review: recent advances in biohydrogenation of unsaturated fatty acids within the rumen microbial ecosystem. J Anim Sci 86, 397-412.

37. Beam TM, Jenkins TC, Moate PJ, et al. (2000) Effects of amount and source of fat on the rates of lipolysis and biohydrogenation of fatty acids in ruminal contents. J Dairy Sci 83, 2564-2573.

38. Gerson T, John A \& King ASD (1985) The effects of dietary starch and fibre on the in vitro rates of lipolysis and hydrogenation by sheep rumen digesta. J Agric Sci (Camb) 105, 27-30.

39. Bauchart D, Legay-Carmier F, Doreau M, et al. (1990) Lipid metabolism of liquid associated and solid-adherent bacteria in rumen contents of dairy cows offered lipidsupplemented diets. Br J Nutr 63, 563-578.

40. Jouany J-P, Lassalas B, Doreau M, et al. (2007) Dynamic features of the rumen metabolism of linoleic acid, linolenic acid and linseed oil measured in vitro. Lipids 42, 351-360.

41. Honkanen AM, Griinari JM, Vanhatalo A, et al. (2012) Characterization of the disappearance and formation of biohydrogenation intermediates during incubations of linoleic acid with rumen fluid in vitro. J Dairy Sci 95, 1376-1394.

42. Shingfield KJ, Ahvenjärvi S, Toivonen V, et al. (2008) Effect of incremental levels of sunflower-seed oil in the diet on ruminal lipid metabolism in lactating cows. Br J Nutr 99 , 971-983.

43. Sackmann R, Duckett SK, Gillis MH, et al. (2003) Effects of forage and sunflower oil levels on ruminal biohydrogenation of fatty acids and conjugated linoleic acid formation in beef steers fed finishing diets. J Anim Sci 81, 3174-3181.

44. Harfoot CG \& Hazlewood GP (1997) Lipid metabolism in the rumen. In The Rumen Microbial Ecosystem, pp. 382-426 [PN Hobson and CS Stewart, editors]. London: Chapman \& Hall.

45. Fuentes MC, Calsamiglia S, Cardozo PW, et al. (2009) Effect of $\mathrm{pH}$ and level of concentrate in the diet on the production of biohydrogenation intermediates in a dual-flow continuous culture. J Dairy Sci 92, 4456-4466. 
46. Zened A, Enjalbert F, Nicot MC, et al. (2013) Starch plus sunflower oil addition to the diet of dry dairy cows results in a trans-11 to trans-10 shift of biohydrogenation. J Dairy Sci 96, 451-459.

47. McKain N, Shingfield KJ \& Wallace RJ (2010) Metabolism of conjugated linoleic acids and 18:1 fatty acids by ruminal bacteria: products and mechanisms. Microbiology 156, 579-588.

48. Lock AL, Tyburczy C, Dwyer DA, et al. (2007) Trans-10 octadecenoic acid does not reduce milk fat synthesis in dairy cows. J Nutr 137, 71-76.

49. Kadegowda AKG, Piperova LS \& Erdman RA (2008) Principal component and multivariate analysis of milk long-chain fatty acid composition during diet-induced milk fat depression. J Dairy Sci 91, 749-759.

50. Shingfield KJ, Sæbø A, Sæbø P-C, et al. (2009) Effect of abomasal infusions of a mixture of octadecenoic acids on milk fat synthesis in lactating cows. J Dairy Sci 92, 4317-4329.
51. Alves SP \& Bessa RJB (2014) The trans-10, cis-15 18:2: a missing intermediate of trans-10 shifted rumen biohydrogenation pathway? Lipids 49, 527-541.

52. Vlaeminck B, Fievez V, Cabrita ARJ, et al. (2006) Factors affecting odd- and branched-chain fatty acids in milk: a review. Anim Feed Sci Technol 131, 389-417.

53. Fievez V, Colman E, Castro-Montoya JM, et al. (2012) Milk odd- and branched-chain fatty acids as biomarkers of rumen function - an update. Anim Feed Sci Technol $\mathbf{1 7 2}$, 51-65.

54. Pantoja J, Firkins JL, Eastridge ML, et al. (1996) Fatty acid digestion in lactating dairy cows fed fats varying in degree of saturation and different fibre sources. J Dairy Sci 79, 575-584.

55. Sjaunja LO, Baevre L, Junkkarinen L, et al. (1990) A Nordic proposal for an energy corrected milk (ECM) formula. Proceedings of the 27th Biennial Session of the International Committee for Animal Recording (ICAR), Paris, 2-6 July 1990. p. 156 